\title{
Torsion hypersurfaces on abelian schemes and Betti coordinates
}

\author{
P. Corvaja, D. W. Masser and U. Zannier
}

Departement Mathematik und Informatik

Preprint No. 2017-17

Fachbereich Mathematik

December 2017

Universität Basel

CH-4051 Basel

$\underline{\text { www.math.unibas.ch }}$ 


\title{
TORSION HYPERSURFACES ON ABELIAN SCHEMES AND BETTI COORDINATES
}

\author{
Pietro Corvaja, David Masser, Umberto Zannier
}

December 22, 2017

\begin{abstract}
In this paper we extend to arbitrary complex coefficients certain finiteness results on Unlikely intersections linked to torsion in abelian surface schemes over a curve, which have been recently proved for the case of algebraic coefficients; in this way we complete the solution of Zilber-Pink conjecture for abelian surface schemes over a curve. As experience has shown also in previous cases, the extension from algebraic to complex coefficients often requires entirely new arguments, whereas simple specialization arguments fail.

The outcome gives as a byproduct new finiteness results when the base of the scheme has arbitrary dimension; another consequence is a proof of an expectation of Mazur concerning the structure of the locus in the base when a given section is torsion. Finally, we show the link with an old work of Griffiths and Harris on a higher dimensional extension of a theorem of Poncelet.
\end{abstract}

\section{Introduction}

A special case of a conjecture of Pink, in the context of unlikely intersections, states the following:

Let $\mathcal{A} \rightarrow \mathcal{C}$ be an abelian-surface-scheme over a complex curve $\mathcal{C}$ and let $\sigma: \mathcal{C} \rightarrow \mathcal{A}$ be a section whose image is not contained in a proper group subscheme. Then there are only finitely many points $x \in \mathcal{C}$ such that $\sigma(x)$ is torsion on the fiber $\mathcal{A}_{x}$.

When the field of definition of the scheme is $\overline{\mathbb{Q}}$, this conjecture has been confirmed in a series of papers [13], [14], [16], dealing respectively with the case where $\mathcal{A}$ is isogenous to a square, or a product of elliptic curves, or is simple (meaning that the generic fibre is geometrically simple). The methods heavily used the arithmetic of $\overline{\mathbb{Q}}$ and the problem arose to obtain the same results over $\mathbb{C}$. One natural approach was by specialization. 
However this turned out to be much more complicated then it was hoped and expected. This difficulty is due to the non-compactness of the base, so that all algebraic specializations in principle might turn out to be bad (see the example in section where we sketch our strategy). ${ }^{1}$

Indeed, the result over $\mathbb{C}$ has been achieved at the moment only for the split cases, where it occupies a substantial part of the papers [13], [14].

A first task of this paper is to settle the remaining important case of a simple $\mathcal{A}$ defined over an arbitrary field of characteristic zero, by means of a different method. Together with the cited results, this completes the proof of the above statement, i.e. the conjecture of Pink in the case of relative dimension 2 over a curve.

This method will yield simultaneously also the solution of the variant of the above conjecture where now $\mathcal{A} \rightarrow S$ is an abelian-surface-scheme over an arbitrary complex variety $S$, and we wish to describe the algebraic hypersurfaces on $S$ where $\sigma$ restricts to a torsion section.

We shall settle completely this issue. In Appendix B we shall briefly treat by a quicker method the case where $\mathcal{A}$ is the product of the Legendre schemes $E_{\lambda}, E_{\mu}$ over the $(\lambda, \mu)$ plane. In particular this will indicate a simplified proof of the transition from $\overline{\mathbb{Q}}$ to $\mathbb{C}$ carried out in the papers [13], [14].

In Appendix $\mathrm{C}$ we shall apply our result to settle an issue raised by B. Mazur (which we describe at the end of the introduction).

For several reasons, we have decided to focus explicitly in this paper on the case of relative dimension 2: this is both for the sake of simplicity and also since the complete proof of Pink conjecture concerns this case. However, we stress that this limitation is by no means essential (and actually the algebraic case has already been carried out for arbitray relative dimension in a paper to be soon submitted).

We now give some statements to put on precise grounds all of this.

Let $S$ be a complex irreducible quasi-projective algebraic variety, let $\pi: \mathcal{A} \rightarrow S$ be a scheme in abelian surfaces and let $\sigma: S \rightarrow \mathcal{A}$ be a section, so $\pi \circ \sigma=i d_{S}$.

As usual, we denote by $\mathcal{A}_{s}$, for $s \in S$, the fibre $\pi^{-1}(s)$. We may assume that the variety $S$ is smooth and we may complete it to a smooth projective one $\bar{S}$ such that $\bar{S} \backslash S$ is a union of hypersurfaces; up to removing if necessary further hypersurfaces, we suppose that $S$ is affine and we recall that $\mathcal{A}_{s}$ is always an abelian variety.

We say that an irreducible hypersurface $X \subset S$ is torsion for $\sigma$ if the restriction of the section $\sigma$ to $X$ is torsion. This amounts to the existence of an integer $n>0$ such that

\footnotetext{
${ }^{1}$ In principle, as pointed out by the referee, the strategy in the case of a compact base (without bad reduction) becomes much simpler, but still some problems remain, e.g. the preservation of simplicty under restriction to curves.
} 
$n \sigma(x)=0$ in the fiber $\mathcal{A}_{x}$ for all $x \in X$.

Our first theorem is

THEOREM 0.1. Let $\mathcal{A} \rightarrow S$ be a scheme in abelian surfaces, over a complex irreducible quasi-projective variety $S$. Let $\sigma: S \rightarrow \mathcal{A}$ be a section. Then there exist only finitely many torsion hypersurfaces in $S$, unless $\sigma(S)$ is contained in a proper subgroup scheme.

Theorem 0.1 follows from [13], [14] when the abelian scheme $\mathcal{A} \rightarrow S$ is not simple. Hence in the sequel of this work (apart from Appendix B) we shall deal with a simple abelian scheme. In this case, $\sigma(S)$ cannot be contained in a proper subgroup scheme, unless it is torsion.

We shall also assume that the scheme $\mathcal{A} \rightarrow S$ is not iso-constant: otherwise one may first assume that $A_{s}$ is constantly equal to the abelian variety $A$. Let us consider the section $\sigma: S \rightarrow A$ and its image $B=\sigma(S) \subset A$. Now, $B$ cannot contain any torsion curve because $A$ is simple. Then each torsion hypersurface $X$ would be sent by $\sigma$ to a point. Then $X$ would lie in the proper algebraic subvariety of $S$ where the fibers of $\sigma$ have dimension higher than the generic one. This gives finiteness.

As anticipated, this result completes the solution of Pink's conjecture for abelian schemes of relative dimension two over a curve (which was provided in [16] only over number fields). Namely, chosing a curve for the base $S$, we obtain:

Theorem 0.2. Let $\mathcal{A} \rightarrow \mathcal{C}$ be an abelian-surface-scheme over a complex curve $\mathcal{C}$ and let $\sigma: \mathcal{C} \rightarrow \mathcal{A}$ be a section whose image is not contained in a proper group subscheme. Then there are only finitely many points $x \in \mathcal{C}$ such that $\sigma(x)$ is torsion on the fiber $\mathcal{A}_{x}$.

It will appear that also Theorem 0.1 follows easily from Theorem 0.2.

Mazur's issue. The problem that we are going to describe was raised by B. Mazur after a talk of one of us. Take a non-torsion section over the whole moduli space of jacobians of curves (canonically polarized) of genus two, which has dimension three. We expect:

(i) Finiteness of torsion surfaces if the section is not torsion. This follows from Theorem 0.1 since the generic jacobian is simple.

(ii) Infinitely many torsion curves. This and more will be proved in Appendix C; it could also be proved, in greater generality, by other methods (general results in this direction will be published in a separate paper with Y. André).

(iii) Mazur's question: do the infinitely many curves mentioned in (ii) all lie in finitely many hypersurfaces? In Appendix $\mathrm{C}$ we shall prove that, as expected by Mazur, the answer is negative: in fact, the torsion curves are dense in the base. 
The talk which prompted this question dealt actually with a specific section related to Pell's equation (see [16]). The question (iii) was especially relevant, because an affirmative answer to it would trivialize many of the finiteness theorems proved in [16].

Very recently, we came across Griffiths and Harris' paper [7], which considers an analogue of a geometrical procedure introduced by Poncelet on pairs of conic.

We recall Poncelet's construction: given two conics $C, C^{\prime}$ on the plane, in general position, one starts with one point $P_{1} \in C$ and a line $l_{1}$ containing $P_{1}$ and tangent to $C^{\prime}$. This pair produces another pair $\left(P_{2}, l_{1}\right)$ by replacing $P_{1}$ with the second intersection point of $l_{1}$ with $C$, and still another pair $\left(P_{2}, l_{2}\right)$ by replacing $l_{1}$ with the second tangent line to $C^{\prime}$ passing through $P_{2}$. Hence we have defined a map $\left(P_{1}, l_{1}\right) \mapsto\left(P_{2}, l_{2}\right)$; this map can be viewed as an automorphism of the curve $X$ formed by the pairs $(P, l)$ where $P \in C$, $l$ is a line tangent to $C^{\prime}$ and $P \in l$. Such a curve turns out to be of genus one, and the automorphism so defined is given by a translation on its jacobian.

It follows that, as proved by Poncelet, the fact that the procedure ends or not only depends on the pair of conics, not on the initial point. We can then consider the set of pairs of conics, possibly up to projective automorphisms (then a two-dimensional space), for which the 'game' ends in $n$ steps. This corresponds to a torsion sub-variety for an elliptic scheme.

In [7] Griffiths and Harris consider the higher dimensional case, where a sequence of tangent planes to a pair of quadrics is constructed by a similar procedure. Varying the quadrics yields a section of a doubly elliptic scheme over a higher dimensional base.

The assertions in [7] (in particular, the last assertion at p. 159) correspond, for the special section which arises, to some of our assertions. Their methods do not refer at all to the notion of Betti map recalled below and havily used by us, and use only basic classical geometry in an elegant fashion.

It seems to us that the present paper (especially the Appendix devoted to Mazur's issue) completes the proof of their assertions.

Our method shall involve a study of the so-called Betti coordinates of an abelian logarithm of a section (a terminology seemingly introduced by D. Bertrand). They are (locally) the coordinates of the abelian logarithm with respect to a locally analytic basis of periods. This seems an interesting matter in its own, which recently attracted some interest from differenty authors and opens several questions that we shall discuss in future. In a recent work by C. Voisin [23], the author implicitely used the Betti map in order to prove the density of torsion points in certain abelian schemes, arising from Lagrangian fibrations.

Indeed, the 'Betti map' is very useful for understanding also the distribution of 'gen- 
eral' torsion varieties associated to a given section. Note that the torsion varieties are the irreducible components of the fibers of rational values of the Betti map.

By means of the results of this paper we shall prove in particular that there are only finitely many torsion hypersurfaces provided the relative dimension is two and the natural assumptions are provided.

Acknowledgments. We express our warmest thanks to an extremely careful referee who detected several inaccuracies, and helped us very much in improving our presentation. The referee also indicated tentative thoughts for later works, for instance suggesting the investigation of the relation between the ranks of the Betti and Kodaira-Spencer maps associated to an abelian scheme with a section. Indeed, a joint work in progress with Y. André explores the role of the Kodaira-Spencer map in this setting. Further warm thanks go to D. Bertrand for his interest and comments which helped our presentation. We are also grateful to C. Voisin for sending us her recent preprint [23] and for a helpful correspondence with the third author.

\section{Preliminaries on Betti coordinates}

\section{$1.1 \quad$ Periods}

We may suppose that $S$ is complex affine and smooth and denote by $d$ its dimension; we let $\bar{S}$ be its closure in some ambient projective space. We can find a finite covering $U_{\alpha}$ by open sets for the complex topology, where $U_{\alpha}, U_{\alpha}^{\prime}$ are simply connected open subsets of $S$ and $\bar{S}$ respectively, so that the topological closure $\overline{U_{\alpha}} \cap S$ of $U_{\alpha}$ in $S$ is contained in $U_{\alpha}^{\prime}$. We may also suppose that each $U_{\alpha}$ is bioholomorphic to an open connected subset of $\mathbb{C}^{d}$.

Above each point $s \in S$ we have a tangent space to $\mathcal{A}_{s}$ at the origin; we thus obtain a holomorphic vector bundle over $S$; refining the coverings $U_{\alpha}$ (resp. $U_{\alpha}^{\prime}$ ) we may suppose that this bundle is trivial over every $U_{\alpha}^{\prime} \cap S$, i.e. isomorphic to $U_{\alpha}^{\prime} \times \mathbb{C}^{2}$.

We have exponential maps $\mathcal{E}_{\alpha}: U_{\alpha}^{\prime} \times \mathbb{C}^{2} \rightarrow \pi^{-1}\left(U_{\alpha}^{\prime}\right)$ which are surjective group homomorphisms on each fiber of $\pi$.

For each $s \in U_{\alpha}^{\prime}$, the kernel of $\mathcal{E}_{\alpha}$ restricted to the fiber over $s$ is a lattice $\Lambda_{\alpha, s}$ of rank 4. Since $U_{\alpha}^{\prime}$ is simply connected, we can find holomorphic functions $F_{\alpha, i}: U_{\alpha} \rightarrow \mathbb{C}^{2}$, $i=1, \ldots, 4$, such that $F_{\alpha, i}(s), i=1, \ldots, 4$, is a basis for $\Lambda_{\alpha, s}$ for each $s \in U_{\alpha}^{\prime}$. In particular, the values of these functions at each point $s \in U_{\alpha}$ are linearly independent over $\mathbb{R}$.

On the intersections $U_{\alpha} \cap U_{\beta}$, we have holomorphic transition functions $G_{\alpha, \beta}: U_{\alpha} \cap$ $U_{\beta} \rightarrow \mathrm{G} L_{2}(\mathbb{C})$ such that $\mathcal{E}_{\beta}(s, v)=\mathcal{E}_{\alpha}\left(s, G_{\alpha, \beta}(s) \cdot v\right)$, for $v \in \mathbb{C}^{2}$. In this notation, the vector $F_{\alpha, i}(s) \in \mathbb{C}^{2}$ corresponds to a tangent vector at the origin of $\mathcal{A}_{s}$ which is expressed 
by the vector $G_{\alpha, \beta}(s) \cdot F_{\alpha, i}(s)$ in the complex plane $\mathbb{C}^{2}$ associated to $\beta$. This last vector is a linear combination with integral coefficients of the basis $F_{\beta, 1}(s), \ldots, F_{\beta, 4}(s)$. We derive the transformation rule

$$
G_{\alpha, \beta}(s) F_{\alpha, i}(s)=\sum_{j=1}^{4} c_{i, j}(\alpha, \beta) F_{\beta, j}(s),
$$

where the matrix $\left(c_{i, j}\right)_{i, j} \in \mathrm{G} L_{4}(\mathbb{Z})$ is locally constant and expresses the monodromy of these functions. ${ }^{2}$

Example. An example in which the base $S$ is the curve $\mathbb{P}_{1} \backslash\{0,1, \infty\}$ is provided by the Legendre scheme $\mathcal{L}$ : for a $\lambda \in \mathbb{P}_{1} \backslash\{0,1, \infty\}$ the fiber $\mathcal{L}_{\lambda}$ is defined by the equation in $\mathbb{P}_{2}$ :

$$
Z Y^{2}=X(X-Z)(X-\lambda Z)
$$

Thus $\mathcal{L}$ is embedded in $\left(\mathbb{P}_{1} \backslash\{0,1, \infty\}\right) \times \mathbb{P}_{2}$. For instance, for $\lambda$ in the region $|\lambda|<$ $1 \&|1-\lambda|<1$, a suitable basis of periods is given by $\pi F(\lambda), \pi i F(1-\lambda)$, where $F(\lambda)=$ $\sum_{m=0}^{\infty} \frac{(m !)^{2}}{2^{4 m}(m !)^{4}} \lambda^{m}$, is a well-known hypergeometric function. Analytic continuation of these functions on a covering of $\mathbb{P}_{1} \backslash\{0,1, \infty\}$ is possible and yields the transformation rules considered above.

Abelian logarithms. Given a regular section $\sigma: S \rightarrow \mathcal{A}$ we can define its abelian logarithm with respect to the above covering: since each $U_{\alpha}^{\prime}$ is simply connected, we can lift the map $\sigma: U_{\alpha}^{\prime} \rightarrow \mathcal{A}$ to a holomorphic map $\left(\mathrm{id} \times L_{\alpha}\right): U_{\alpha}^{\prime} \rightarrow U_{\alpha}^{\prime} \times \mathbb{C}^{2}$. So on each $U_{\alpha}^{\prime}$ we obtain a holomorphic function $L_{\alpha}$ such that

$$
\mathcal{E}_{\alpha}\left(s, L_{\alpha}(s)\right)=\sigma(s)
$$

This $L_{\alpha}$ is unique up to the addition of a linear combination with integral coefficients, constant in $U_{\alpha}^{\prime}$, of the functions $F_{\alpha, 1}, \ldots, F_{\alpha, 4}$.

As above, on the intersections $U_{\alpha} \cap U_{\beta}$ we have holomorphic transitions functions $G_{\alpha, \beta}: U_{\alpha} \cap U_{\beta} \rightarrow G L_{2}(\mathbb{C})$ such that $\mathcal{E}_{\beta}(s, v)=\mathcal{E}_{\alpha}\left(s, G_{\alpha, \beta}(s) \cdot v\right)$. Using this transition (with $v=L_{\alpha}$ ), we obtain corresponding transition functions for the logarithms, up to the already mentioned translation by lattice elements.

Betti coordinates. Following a terminology introduced seemingly by D. Bertrand, we now define Betti coordinates of an abelian logarithm of a section. Given an algebraic section $\sigma$ as above and keeping the above data for the covering, trivialization, period

\footnotetext{
${ }^{2}$ On the contrary the transition functions $G_{\alpha, \beta}$ do not involve monodromy of periods but merely the structure of the vector bundle; actually, if the vector bundle is trivial over $S$, we we could choose these transition functions to be the identity.
} 
basis and abelian logarithm, we express this last one in terms of the period basis:

$$
L_{\alpha}(s)=\sum_{i=1}^{4} b_{\alpha, i}(s) F_{\alpha, i}(s)
$$

where the coefficients $b_{\alpha, i}$ are real valued functions of $s \in U_{\alpha}$. (In this notation, we shall often omit the reference to $U_{\alpha}^{\prime}$ when this causes no confusion.) We also define the Betti map

$$
\mathfrak{b}(s)=\mathfrak{b}_{\alpha}(s)=\left(b_{\alpha, 1}(s), \ldots, b_{\alpha, 4}(s)\right) .
$$

These coordinate functions are easily seen to be real-analytic. Of course, on changing the open set $U_{\alpha}$ to $U_{\beta}$, these Betti coordinates obey transformation rules corresponding to the ones mentioned above.

\subsection{About the differential of the Betti map.}

In the logic of our proof a central issue will be the differential of the Betti map.

Actually, by considering in details the rank of this differential, the proof could be made more self-contained at the cost of increasing its length. (See remarked in 2.14 and section 2.6.)

In any case we collect in this section some relevant facts about the rank of this differential.

This rank is defined as the rank of the jacobian matrix of these Betti coordinates, at a certain point of $S$, with respect to any choice of local real-coordinates $x_{j}, y_{j}$, where we can assume for instance that $z_{j}=x_{j}+i y_{j}$ on $U_{\alpha}$ are holomorphic coordinates on $U_{\alpha}$ and $x_{j}, y_{j}$ are the corresponding real and imaginary parts.

We observe that this rank is well defined, i.e. it is independent of the open set $U_{\alpha}$ and of the choice of both the periods and the abelian logarithms (which are determined up to integer translation). We call generic rank the maximal rank of this differential on $S$. The set where the rank decreases is a (proper) closed real-analytic subvariety; hence the set where the rank is the generic one is open and dense (since $S$ is connected).

Let $s_{0}$ be a point where $\operatorname{rank}(\mathrm{d} \mathfrak{b})_{s_{0}}=r$ is maximal. Then by a version of the implicit function theorem, the fiber $\mathfrak{b}^{-1}(\mathfrak{b}(s))$ is, in a neighborhood of $s_{0}$, a real analytic variety of dimension $2 d-r$.

We observe an easy but relevant fact. Before stating this, recall that the tangent space at a point on a complex variety is understood to be a complex vector space. Viewing the variety as a real manifold, we can see the same tangent space as a real vector space, having a complex structure induced by an operator $J$ with $J^{2}=-I$; then the complex subspaces are those real vector subspaces invariant by $J$. 
We have:

Proposition 1.1. The kernel of this jacobian matrix at any given point of $S$ is a complex vector space. Also, the fibers of the Betti map $s \mapsto\left(b_{1}(s), \ldots, b_{4}(s)\right)$ are complex subvarieties.

PROOF. For this argument we work locally on some $U_{\alpha}$, identifying it with an open subset of $\mathbb{C}^{d}$, by means of the mentioned biholomorphism.

We have locally the equation $L(s)=\sum_{i=1}^{4} b_{i}(s) F_{i}(s)$, where we have omitted the reference to $\alpha$, and where what matters for us is that $L(s), F_{i}(s)$ are holomorphic functions $U_{\alpha} \rightarrow \mathbb{C}^{2}$, with $F_{i}(s)$ linearly independent over $\mathbb{R}$ for each $s \in U_{\alpha}$ and where $b_{i}$ are realvalued differentiable functions. Then, taking the complex conjugates we obtain also the relations $\overline{L(s)}=\sum_{i=1}^{4} b_{i}(s) \overline{F_{i}(s)}$. In view of the fact that the $f_{i}(s)$ are linearly independent over $\mathbb{R}$ (for each $s$ ), the $4 \times 4$ matrix obtained from the $F_{i}(s)$ and $\overline{F_{i}(s)}$ is invertible, and this uniquely determines the $b_{i}(s)$ (and one may express them by Cramer's rule).

Let $s_{0} \in S$ and let $v_{0} \in \mathbb{C}^{d}$ be a vector in the kernel of the jacobian map at $s_{0}$; here we view $v_{0}$ as a vector in $\mathbb{R}^{2 d}$ with respect to the previously defined real and imaginary parts. By definition, the functions $\mathbb{R} \ni t \rightarrow b_{i}\left(s_{0}+t v_{0}\right)$ have vanishing derivative at $t=0$, hence the function $G(s):=L(s)-\sum_{i=1}^{4} b_{i}\left(s_{0}\right) F_{i}(s)$ is $O\left(t^{2}\right)$ on the real line $s=s_{0}+t v_{0}$, for real $t$ tending to 0 . However the function $G$ is holomorphic, hence the same estimate must hold on the complex line $s=s_{0}+t v_{0}, t$ complex near the origin. Hence for a complex multiple $v_{1}$ of $v_{0}$, we have, using the definition of $L(s)$, that

$$
\sum_{i=1}^{4}\left(b_{i}(s)-b_{i}\left(s_{0}\right)\right) F_{i}(s)=O\left(t^{2}\right)
$$

for $s=s_{0}+t v_{1}$ and again for real $t \rightarrow 0$. On the other hand, since the determinant of the mentioned $4 \times 4$ matrix is non-zero for every $s$, its absolute value is bounded from below in a neighborhood of $s_{0}$. Inverting that matrix we then obtained a bounded matrix in such a neighborhood. This yields $\left(b_{i}(s)-b_{i}\left(s_{0}\right)\right)=O\left(t^{2}\right)$ on that line, i.e. $v_{1}$ lies in the kernel, proving the first part of the proposition.

The second part essentially follows from the above, but we can prove it directly, in a similar way: given $\left(c_{1}, \ldots, c_{4}\right) \in \mathbb{R}^{4}$ the corresponding level subvariety for the map $\left(b_{1}, \ldots, b_{4}\right)$ is also defined by the holomorphic equation $L(s)=\sum_{i=1}^{4} c_{i} F_{i}(s)$.

Note that this also proves the first part at points where the Jacobian rank is constant in a neighborhood. Indeed, in this case the relevant point must be smooth and the tangent space to the fiber is exactly the kernel of the jacobian matrix.

Proposition 1.2. For every $k \in \mathbb{N}$, the set of $s \in S$ such that the dimension at $s$ of $\mathfrak{b}^{-1}(\mathfrak{b}(s))$ is $\geq k$ is a closed set. In particular all the fibers of the Betti map have real dimension $\geq 2 d-r$, where $r$ is the generic rank. 
Before the proof we remark that this is not entirely automatic since we are dealing with real analytic maps. (For instance consider the real analytic map $(x, y) \mapsto x^{2}+y^{2}$ near the origin.)

Proof. Consider in $U_{\alpha} \times \mathbb{C}^{4}$ the complex variety $X$ defined by $L(s)=\sum_{i=1}^{4} w_{i} F_{i}(s)$, where we omit the reference to $\alpha$. We have a projection map $\varphi: X \rightarrow \mathbb{C}^{4}$. By the Theorem of Cartan-Remmert, see e.g. p. 271 in [10], for each $k \in \mathbb{N}$, the set $X_{k}$ of the points $x=(s, \mathbf{w}) \in X$ such that $\varphi^{-1}(\varphi(x))$ has local dimension $\geq k$ at $x$, is closed.

Let $\tilde{X}=X \cap\left(U_{\alpha} \times \mathbb{R}^{4}\right)$; this is a closed subset of $X$ and it coincides with the graph of the Betti map. In particular, the intersection $\tilde{X}_{k}:=X_{k} \cap \tilde{X}$ is also a closed subset of the graph $\tilde{X}$ of the Betti map. The projection $\tilde{X} \rightarrow U_{\alpha}$ is a homeomorphism, so the image of $X_{k} \cap \tilde{X}$ in $U_{\alpha}$ is closed in $U_{\alpha}$. This proves the first assertion, since we may work locally in each $U_{\alpha}$.

Since by the above remark the projection to $U_{\alpha}$ of $\tilde{X}_{2 d-r}$ is open and dense in $U_{\alpha}$, the second assertion also follows.

Although it will not be needed in the sequel, for the purpose of clarity and completeness, we shall collect some further facts about the structure of Betti maps.

First, observe that if the section $\sigma$ is torsion, then the corresponding Betti coordinates are constant (equal to rational numbers). It is a consequence of a deep theorem of Manin (see [11] and corrections in [3] and [4]) that a converse holds: if the Betti coordinates are locally constant, then the section is the sum of a torsion section plus a section with image in a constant abelian subvariety.

- One might also ask about the structure of the variety defined by the vanishing of the maximal minors of the jacobian of the Betti coordinates. For this purpose, we can consider again, given locally an open subset $U \subset S$, isomorphic to an open ball of $\mathbb{C}^{d}$, the variety $X \subset U \times \mathbb{C}^{4}$ defined by $L(s)-\sum_{i=1}^{4} z_{i} F_{i}(s)=0$. The variety $X$ is complex analytic of dimension $d+2$. We can consider the projection $\varphi: X \rightarrow \mathbb{C}^{4}$. The locus in which we are presently interested is the intersection with $U \times \mathbb{R}^{4}$ of the locus in $X$ where the differential of $\varphi$ on $X$ has not maximal rank (indeed the only complex subspace of $\mathbb{C}^{4}$ containing $\mathbb{R}^{4}$ is the full space $\mathbb{C}^{4}$ ). This last one is a complex-analytic subvariety of $X$.

We do not know whether the former set of points, i.e. the points of $U$ where the rank of the Jacobian is not maximal, is necessarily a complex analytic subvariety of $S$ and even do not know whether it may have odd real dimension.

Restricting for instance to the case where $S$ is a complex surface with local complex coordinates $z, w$, it may be proved that the following identity holds:

$$
4 D_{F} \operatorname{det} \mathrm{J} a c= \pm|\operatorname{det} M|^{2}
$$


where:

(i) $D_{F}$ is the determinant of the $4 \times 4$-matrix with rows given by the $F_{i}$ and their complex conjugates;

(ii) $M$ is the $2 \times 2$ matrix whose column vectors are $\partial_{z}(L)-\sum_{i} b_{i}(s) \partial_{z} F_{i}, \partial_{w}(L)-$ $\sum_{i} b_{i}(s) \partial_{w} F_{i}$ and where $\partial_{z}, \partial_{w}$ are the complex derivatives with respect to $z$ and $w$.

It may be checked that for instance choosing the vectors $F_{i}$ to be $(1,0),(z, 0),(0,1),(0, w)$ $L(z, w)=\left(z^{2}+w^{2}, z w\right)$ the vanishing set of the jacobian determinant is a real analytic but non complex analytic variety in the region $S \subset \mathbb{C}^{2}$ where $z, w$ are both non-real. This choice would represent an abelian logarithm of a transcendental section; however we suspect that also in the case of algebraic sections the variety in question can be non-analytic.

As to its dimension, seemingly it should be even.

\subsection{Torsion values}

As explained in the introduction, we are interested in the set of points $s \in S$ where $\sigma(s)$ is torsion. More precisely, for each integer $n \geq 0$, we can consider the set $\Sigma_{n}$ of points $s \in S$ where $\sigma(s)$ has exact order $n$. The relation $n \cdot \sigma(s)=0$ is algebraic on $S$, hence $\Sigma_{n}$ is a quasi-projective subset in $S$. Note that saying that $\sigma$ is a non-torsion section amounts precisely to the assertion that no finite union of $\Sigma_{n}$ covers $S$, or equivalently that no $\Sigma_{n}$ is Zariski-dense. Usually, these sets will have codimension $\geq 2$ (because the fibers of $\mathcal{A} \rightarrow S$ have dimension 2). However, it may happen that some $\Sigma_{n}$ has codimension one and this is precisely the main situation investigated in the paper.

We end this section with an important remark about these sets:

Proposition 1.3. Each set $\Sigma_{n}$ is closed in $S$. In particular, for distinct $m, n$ the closures of $\Sigma_{m}$ and $\Sigma_{n}$ in $S$ are disjoint.

We remark that the last conclusion clearly follows from the first.

This conclusion is trivial if $\Sigma_{n}$ consists of isolated points, whereas when the closure of $\Sigma_{n}$ has positive dimension, it amounts to saying that the order of any point in this closed set remains exactly $n$ (whereas a priori the order might decrease to a proper divisor).

Proof. It suffices to argue locally on each $U_{\alpha}$. Now, the vector $\mathfrak{b}_{\alpha}$ of Betti coordinates assumes on $\Sigma_{n}$ rational values with exact common denominator $n$; hence, $\mathfrak{b}_{\alpha}$ takes values on $\Sigma_{n}$ in a discrete closed set. These values will therefore be constant on connected components of $\Sigma_{n}$. Hence $\Sigma_{n}$ is closed.

The proposition can also be proved algebraically just by appealing to the well-known fact that reduction maps (with respect to some maximal ideal) are injective on torsion points when the torsion order is prime to the residue characteristic (see for instance [18] 
Lemma 2 and the following lines for this last fact). We may then deduce the proposition as follows: take any curve $\Gamma$ inside $\Sigma_{n}$ and consider its closure in $S$; the restriction of $\sigma$ to such a closure is a section of exact order (generically) $n$, and specialization at a point is a reduction operation, to which the above applies (this works also for non-smooth points of $\Gamma$ by taking base extension to a normalisation).

\section{Theorem 0.1 over number fields}

In this section we work over an arbitrary variety $S$ defined over a number field $k$; our aim is to prove Theorem 0.1 in this case; in next section we shall deduce the full Theorem 0.1 , where the field of definition is the complex number field, by first reducing to a finitely generated field over $\mathbb{Q}$ and then by using the present result over number field after increasing the dimension of the base. Hence our main result, which shall be proved in this section, is the following:

THEOREM 2.1. Let $S$ be a smooth affine geometrically irreducible variety of dimension $d \geq 1$ defined over a number field $k, \mathcal{A} \rightarrow S$ a simple family of abelian surfaces. Let $\sigma: S \rightarrow \mathcal{A}$ be a non-torsion section. There exist only finitely many torsion hypersurfaces $X \subset S$ for $\sigma$.

Our torsion hypersurfaces will automatically be defined over $k$ and we shall consider their irreducible components over $\bar{k}$.

We shall argue by induction on the dimension of $S$, the case of dimension 1 being covered by [MZ].

\subsection{Sketch of the strategy}

In this sketch, we treat for simplicity only the case when $S$ is a surface, showing how to reduce to curves. We assume there are infinitely many torsion hypersurfaces (i.e. curves) $X_{n}$ and we wish to find a curve $Y \subset S$ defined over $\overline{\mathbb{Q}}$ intersecting infinitely many of the $X_{n}$, so to be able to apply induction. The difficulty is that the $X_{n}$ may 'escape' to infinity, in the sense that for every fixed curve $Y$, only finitely many of them intersect $Y$ in $S$. The following example, with $S=\mathbb{A}^{2}$, shows that this problem cannot be neglected:

Example: There exists a sequence of plane curves $X_{n} \subset \mathbb{A}^{2}$, for $n=1,2 \ldots$, defined over $\overline{\mathbb{Q}}$ such that:

1) $\operatorname{deg} X_{n} \rightarrow \infty$ for $n$ tending to $\infty$;

2) each algebraic curve $Y \subset \mathbb{A}^{2}$ defined over $\overline{\mathbb{Q}}$ intersects in $\mathbb{A}^{2}$ only finitely many curves $X_{n}$. 
To prove that such a sequence indeed exists, let us enumerate the irreducible curves in $\mathbb{A}^{2}$ defined over $\overline{\mathbb{Q}}$ as $Y_{1}, Y_{2}, \ldots$ Each curve $Y_{i} \subset \mathbb{A}^{2}$ will be defined by a polynomial equation $f_{i}(x, y)=0$. Now define $X_{i}$ to be the curve of equation $f_{1}(x, y) \cdots f_{i}(x, y)=1$. Clearly, $Y_{j}$ cannot meet $X_{j}, X_{j+1}, \ldots$ in $\mathbb{A}^{2}$.

To overcome this kind of obstacle, our first point in the strategy will be to find a compact region in $S$ intersecting infinitely many of the $X_{n}$. We shall achieve this by using a uniform version of Silverman's bounded height theorem: namely, by intersecting the $X_{n}$ with a suitable denumerable union of curves of bounded height, we shall find infinitely many intersection points of bounded height and by taking conjugates we shall deduce that some conjugate of each of these points lies in a bounded region. These curves are parte of a continuous algebraic family of curves $\mathcal{C}_{\theta}$, where we shall restrict $\theta$ to roots of unity. By results on uniform distribution and a simple measure theoretic lemma we shall prove the existence of a single $\mathcal{C}_{\theta}$ (where $\theta$ might possibly be transcendental) intersecting infinitely many of the $X_{n}$ in the said bounded region and in $\gg \operatorname{deg} X_{n}$ points.

By means of a rather delicate lower bound of Masser and David (used also in the paper [16], [15]) we shall prove that $\operatorname{deg} X_{n}$ is at least a positive power of $n$. Using this fact, by means of a theorem of Gabrielov in real analytic geometry we shall deduce that the Betti map (restricted to the said bounded region) takes many distinct values on each $\mathcal{C}_{\theta} \cap X_{n}$ and this shall allow us to apply Pila-Wilkie estimates, deducing that the image of the Betti map on $\mathcal{C}_{\theta}$ contains a real algebraic arc. Applying algebraic independence results of André this forces $\mathcal{C}_{\theta}$ to be a torsion curve which is impossible.

This last argument has something in common with the case when $S$ has dimension one, where however the present use of uniform Silverman's bounded height theorem was totally absent.

Another important ingredient of the proof is a specialization theorem of the second author, which shall enable us to perform certain specializatios to algebraic points preserving the simplicity of the abelian fibers.

For the case when the Betti map has maximal rank, a simpler proof is possible.

In this case, our torsion curves $X_{n}$ will lie in the locus $\tilde{E}$ where the rank is $<4$, because the Betti map is locally constant on $X_{n}$, forcing $\tilde{E}$ to have (real) dimension $\leq 3$ (actually exactly 3 , otherwise finiteness follows). Actually, since the Betti map is locally constant on each $X_{n}$, we may restrict to the sub-locus $E$ where the restriction of the Betti map to $\tilde{E}$ has rank $\leq 1$. Now the image of the Betti map on this locus $E$ shall be a finite union of points and sub-analytic smooth arcs.

The image $\mathfrak{b}\left(X_{n}\right)$ will consist of finitely many rational points with the same denomi- 
nator $n .^{3}$

Now, if there are infinitely many accumulation points in the union of these images, we may pick one such point in the interior of one of the mentioned arcs, and choose one of its pre-images $y_{0}$ such that the Betti map restricted to $E$ has rank exactly one at $y_{0}$. If this $y_{0}$ is algebraic, it is easy to find a suitable algebraic curve $Y$ defined over $\overline{\mathbb{Q}}$ passing through $y_{0}$ and intersecting infinitely many $X_{n}$, reducing to the algebraic case. Otherwise, we choose an algebraic curve defined over $\overline{\mathbb{Q}}$, passing sufficiently near to $y_{0}$. Then the image of this curve by $\mathfrak{b}$ will coincide with $\mathfrak{b}(E)$ in a neighbourhood of $y_{0}$, and so will contain infinitely many rational points, as desired. (This will be achieved by local analysis.)

If, on the contrary, there are only finitely many accumulation points, let us fix one of them. We use again the mentioned curves $\mathcal{C}_{\theta}$. We may find infinitely many complex numbers $\theta$ on the circle $S_{1}$ such that the image of the Betti map on $\mathcal{C}_{\theta}$ contains a neighborhood of the said accumulation point. This will enable us to conclude, by finding an algebraic $\theta$ with such property.

\subsection{Auxiliary results}

In this section we collect some preliminary results which will be used in our proofs.

We embed once for all $S$ in a projective space $\mathbb{P}_{N}$, so that by degree and height we will refer to the ones associated to the given embedding.

We use the fact that every abelian variety is isogenous to a principally polarized one (see [22]) and that the statement of Theorem 0.1 is invariant under isogeny. We then assume from now on that the scheme $\mathcal{A} \rightarrow S$ is principally polarized.

The first auxiliary result is the following theorem proved by Masser in [12].

THEOREM 2.2. There exists an effective absolute constant $l$ such that for every real numbers $\delta, h \geq 1$, the set of points $s \in S(\bar{k})$, with $[k(s): k] \leq \delta, h(s) \leq h$ such that the endomorphism ring of $\mathcal{A}_{s}$ is not isomorphic to the generic endomorphism ring, is contained in a hypersurface of $S$ of degree $\ll \max (\delta, h)^{l}$, where the implied constant depends only on $k, S, \mathcal{A}$ and the given embedding.

Observe that for an abelian variety $A$, being simple is equivalent to $\operatorname{End}(A)$ having no zero divisors. Hence, for instance, one obtains from Theorem 2.2 'many' algebraic points on $S$ where the variety $\mathcal{A}_{s}$ is simple. To make this deduction, we can view $S$ as a finite cover of $\mathbb{A}^{d}$, by a morphism of a certain degree $\delta$. Every $k$-rational point in $\mathbb{A}^{d}$ has a pre-image in $S$ of degree $\leq \delta$. Take now a 'large' value of $h$. By Theorem 2.2 the set of points in $\mathbb{A}^{d}(k)$ whose pre-image in $S$ is exceptional, is contained in a hypersurface of

\footnotetext{
${ }^{3}$ Note that the Betti map is not necessarily globally defined on $X_{n}$
} 
$\mathbb{A}^{d}$ of degree bounded by $\ll h^{l}$. In particular, in a given line of $\mathbb{A}^{d}$ there are at most $\ll h^{l}$ exceptional points. Since the total number of $k$-rational points on a given line of height $\leq h$ is $\gg 2^{h}$, we obtain 'many' non-exceptional points, as asserted. In particular, by taking $h$ 'large', we obtain that the non-exceptional points are Zariski dense.

Another useful ingredient will be the following upper bound for the order of of a torsion point on an abelian variety, in term of its degree. The first result in this direction in due to David (in the case of simple abelian varieties); here we use a variant of it, where simplicity for the abelian variety is not required, drawn from [16]:

Proposition 2.3. Given $g \geq 1$ there is a constant $c=c(g)$ with the following property. Let $A$ be a principally polarized abelian variety of dimension $g$ defined over a number field $\kappa$ and let $P$ be a point on $A$ with finite order $n$. Then

$$
n \leq c([\kappa: \mathbf{Q}][\kappa(P): \kappa] \max \{1, h(A)\})^{G}
$$

for $G=8^{g} g !^{2}$ and the semistable Faltings height $h(A)$.

This is Lemma 7.1 in [16].

Further, we shall need an algebraic independence result due to André. We give the formulation appearing in Lemma 5.1 of [16]. Recall that to our section $\sigma: S \rightarrow \mathcal{A}$ is associated locally on $U_{\alpha}$ an abelian logarithm $L: U_{\alpha} \rightarrow \mathbb{C}^{2}$; let us write $L(s)=$ $\left(l_{1}(s), l_{2}(s)\right)$ for two holomorphic functions $l_{1}, l_{2}: U_{\alpha} \rightarrow \mathbb{C}$. Also, the four periods are given by holomorphic maps $F_{i}: U_{\alpha} \rightarrow \mathbb{C}^{2}$, where $F_{i}=\left(f_{i, 1}, f_{i, 2}\right)$, where for $i=1, \ldots, 4$ $f_{i, 1}, f_{i, 2}: U_{\alpha} \rightarrow \mathbb{C}$ are holomorphic functions.

Theorem 2.4 (André). Given a simple family $\mathcal{A} \rightarrow S$ of abelian varieties, non isoconstant, and a non-torsion section $\sigma: S \rightarrow \mathcal{A}$, locally on each open set $U_{\alpha}$ the functions $l_{1}, l_{2}$ are algebraically independent over $\mathbb{C}\left(f_{i, j}\right),(i, j) \in\{1, \ldots, 4\} \times\{1,2\}$.

\subsection{Proofs}

We assume in the sequel that $d \geq 2$. Recall also that we are assuming that $\mathcal{A} \rightarrow S$ is not isoconstant.

We denote by $\mathcal{N}$ the set of integers $n \geq 1$ such that the dimension of the set denoted previously by $\Sigma_{n}$ (the variety of points $p$ in $S$ where $\sigma(p)$ has exact order $n$ ) has dimension $d-1$; our aim is to prove that in fact $\mathcal{N}$ is finite, and we shall assume the contrary, deriving a contradiction.

For $n \in \mathcal{N}$, we denote by $X_{n} \subset S$ the stratum of dimension $(d-1)$ of $\Sigma_{n}$. Note that $X_{n}$ may be reducible; we shall generally denote by $Y_{n}$ an irreducible component of $X_{n}$ over $\bar{k}$. 
In order to prove Theorem 2.1, we start by taking a suitable algebraic family of curves on $\bar{S}$. We can use the following simple lemma, which practically consists in defining a useful system of coordinates:

Lemma 2.5. There exists a dominant rational map $\lambda: \bar{S} \rightarrow \mathbb{A}^{d-1}$ defined over $k$ such that:

(1) the fibers of $\lambda$ are curves and the generic fibers are irreducible;

(2) the indeterminacy locus of $\lambda$ (i.e. the intersection of the closures of the fibers) is contained in $S$, contains a point $s_{0}$ where the abelian variety $\mathcal{A}_{s_{0}}$ is simple and a point $s_{1}$ such that the variety $A_{s_{1}}$ is not isomorphic to $A_{s_{0}}$;

(3) each fiber lies in the numerical class $A^{d-1}$ for an ample divisor $A$ of $\bar{S}$.

(4) for every choice of points $\theta=\left(\theta_{1}, \ldots, \theta_{d-1}\right), \theta^{\prime}=\left(\theta_{1}^{\prime}, \ldots, \theta_{d-1}^{\prime}\right)$ in the image of $\lambda$ such that $\theta_{i} \neq \theta_{i}^{\prime}$ for every $i=1, \ldots, d-1$, the curves $\mathcal{C}_{\theta}$ and $\mathcal{C}_{\theta^{\prime}}$ intersects only in the base-locus, in particular in $S$.

Proof. We start by defining a linear system of curves $\mathcal{C}_{\lambda}$, of dimension $d-1$ as follows. As above, we embed $\bar{S}$ into a projective space $\mathbb{P}_{N}$; denote by $T:=\bar{S} \backslash S$ the hypersurface at infinity of $S$. We take a linear subspace $R_{1}$ of codimension two in $\mathbb{P}_{N}$ intersecting $T$ in a subvariety of dimension $d-3$ (which we interpret as empty if $d=2$ ) and such that $R_{1} \cap S$ contains a point $s_{0}$ such that the abelian variety $\mathcal{A}_{s_{0}}$ is simple (we can do this by applying Theorem 2.2) and a point $s_{1}$ with $A_{s_{1}}$ not isomorphic to $A_{s_{0}}$ (we can do this since we are assuming that the scheme $\mathcal{A} \rightarrow S$ is not isoconstant).

The pencil of hyperplanes containing $R_{1}$ defines a linear system in $S$. By Bertini's theorem we may also choose $R_{1}$ suitably (and defined over $\overline{\mathbb{Q}}$ ) so that the generic section of $S$ by this pencil is geometrically irreducible.

Chosing a parameter $\lambda_{1}$ for this pencil of hyperplanes corresponds to a rational map $\lambda_{1}: S \rightarrow \mathbb{P}_{1}$, restriction to $S$ of a projection $\mathbb{P}_{N} \backslash R_{1} \rightarrow \mathbb{P}_{1}$. The fibers of $\lambda_{1}$ are the hyperplane sections in the linear system. If $d=2$ we stop at this stage. Otherwise we choose another codimension-two subspace $R_{2}$ such that $R_{1} \cap R_{2} \cap T$ has dimension $\leq d-4$, giving rise to another rational map $\lambda_{2}: S \rightarrow \mathbb{A}^{1}$ such that the generic fiber for the map $\left(\lambda_{1}, \lambda_{2}\right): S \rightarrow \mathbb{A}^{2}$ is also geometrically irreducible. We can also suppose that $R_{2}$ contains the point $s_{0}$. We continue until we obtain a map $\lambda=\left(\lambda_{1}: \ldots: \lambda_{d-1}\right): S \rightarrow \mathbb{A}^{d-1}$, whose fibers are curves $\mathcal{C}_{\theta}, \theta \in \mathbb{A}^{d-2}$, generically geometrically irreducible. Also, all of these curves contain the chosen point $s_{0}$ above which $\mathcal{A}$ is simple.

Moreover, according to the above construction, if $\left(\theta_{1}, \ldots, \theta_{d-1}\right)$ and $\left(\theta_{1}^{\prime}, \ldots, \theta_{d-1}^{\prime}\right)$ are points in the image of $\lambda$ such that $\theta_{i} \neq \theta_{i}^{\prime}$ for each index $i=1, \ldots, d-1$, then $\mathcal{C}_{\theta}$ and $\mathcal{C}_{\theta^{\prime}}$ meet only in the base locus and in particular do not meet at infinity.

In the sequel we shall always denote by $\mathcal{C}_{\theta}$, for $\theta=\left(\theta_{1}, \ldots, \theta_{d-1}\right) \in \mathbb{A}^{d-1}$, the fiber $\lambda^{-1}(\theta)$ in $\bar{S}$ (so $\mathcal{C}_{\theta}$ is a complete curve). By our construction, the $\mathcal{C}_{\theta}$ are in particular 
algebraically equivalent, and for each hypersurface $Y \subset \bar{S}$, the degree of $Y$ (in $\mathbb{P}_{N}$ ) equals the intersection product $Y \cdot \mathcal{C}_{\theta}$. Also, by property (2) in Lemma 2.5, the abelian scheme restricted above $\mathcal{C} \theta$ is simple and not isoconstant.

We start our proof of Theorem 2.1 by the following weak version of it:

Lemma 2.6. There exist only finitely many torsion hypersurfaces of given degree.

Here, we are considering irreducible components over $\bar{k}$.

Proof. Let by contradiction $Y_{n} \subset X_{n}, n \in \mathcal{N} \subset\{0,1,2 \ldots\}$, run through an infinite sequence of torsion hypersurfaces, defined over $\bar{k}$ and irreducible, such that their closure $\overline{Y_{n}}$ has fixed degree $D_{1}$. By our present notation, for each $n \in \mathcal{N}, \sigma$ has exact order $n$ on $Y_{n}$.

Then $\overline{Y_{n}} \cap T$ is a hypersurface of $T$ of degree $D_{2}=D_{1} \cdot \operatorname{deg} T$. The family of hypersurfaces of $T$ of degree $D_{2}$ is finite dimensional; it is parametrized by a finite union of projective spaces (possibly of different dimensions). Let now $\theta^{(1)} \in \mathbb{P}_{d-1}(k)$ be a $k$-rational point and consider the curve $\mathcal{C}_{\theta^{(1)}}$; it has only finitely many points in $T$. For each $n$ in the infinite set $\mathcal{N}$ the intersection product $\mathcal{C}_{\theta} \cdot \overline{Y_{n}}=D_{1}$ is the degree of $Y_{n}$, so it is $>0$. Suppose that for infinitely many $n \in \mathcal{N}$, at least one point of intersection $\overline{Y_{n}} \cap \mathcal{C}_{\theta^{(1)}}$ lies in $S$, i.e. outside $T$; then $\mathcal{C}_{\theta^{(1)}}$ is not a torsion curve (because then by Proposition 1.3 the order of torsion would be constant, i.e. not depending on $n$, so $\mathcal{C}_{\theta^{(1)}}$ could intersect only one $Y_{n}$ ) and we can apply [16] and conclude.

Suppose on the contrary that all but finitely many $\overline{Y_{n}}$ intersect $\mathcal{C}_{\theta^{(1)}}$ only at infinity (i.e. in $T$ ). Then in particular for each $n$ in an infinite set $\mathcal{N}_{1} \subset \mathcal{N}$ the variety $Y_{n} \cap T$ passes through one of the finitely many points at infinity of $\mathcal{C}_{\theta^{(1)}}$. This means that the varieties $Z_{n}=Y_{n} \cap T$, for $n \in \mathcal{N}_{1}$, are contained in the family of hypersurfaces of $T$ of degree $D_{2}$ passing through one of the points of $\mathcal{C}_{\theta^{(1)}} \cap T$. Let us call $\Lambda_{1}$ such a family, which will be prametrized by a finite union of projective spaces. For a generic point $p$ of $T$, the sub-family formed by those hypersurfaces of degree $D_{2}$ passing through $p$ has a strictly less dimension than the full family. Hence we can find a second curve $\mathcal{C}_{\theta^{(2)}}$, for a rational point $\theta^{(2)} \in \mathbb{P}_{d-1}(k)$, such that the varieties belonging to $\Lambda_{1}$ intersecting $\mathcal{C}_{\theta^{(2)}}$ form a subfamily $\Lambda_{2}$ of stricly lower dimension than $\Lambda_{1}$. If $\mathcal{C}_{\theta^{(2)}}$ intersects infinitely many $Y_{n}$ in $S$, we are done as before. Otherwise there exists an infinite set $\mathcal{N}_{2}$ of indices such that for each $n \in \mathcal{N}_{2}$ both the intersections $Y_{n} \cap \mathcal{C}_{\theta^{(1)}}$ and $\overline{Y_{n}} \cap \mathcal{C}_{\theta^{(2)}}$ lie entirely at infinity. But this means that $Z_{n}=\overline{Y_{n}} \cap T$ lies in the lower dimensional family $\Lambda_{2}$.

Going ahead, we can find finitely many curves $\mathcal{C}_{\theta^{(1)}}, \ldots, \mathcal{C}_{\theta^{(N)}}$, defined over $k$, such that no hypersurface of degree $D_{2}$ in $T$ can intersect each of the finite sets $\mathcal{C}_{\theta^{(1)}} \cap T$, for $i=1, \ldots, N$; in other words, no hypersurface of degree $D_{1}$ in $\bar{S}$ can intersect each such 
curve only at infinity. So one of these curves $\mathcal{C}_{\theta^{(i)}}$ intersects infinitely many $Y_{n}$ in $S$ and we apply [16] as previously explained.

Remark. In general, the variety of points of $S$ of exact order $n$ might be irreducible over $k$, but reducible over $\bar{k}$, and of course its geometrically irreducible components would have lower degree. Lemma 2.6 just proved states that even the degree of these components tend to infinity with $n$.

Suppose now we dispose of an infinite sequence of torsion hypersurfaces $X_{n}, n \in \mathcal{N}$, of degree tending to infinity. Here $\mathcal{N} \subset\{1,2, \ldots\}$ is an infinite set and $X_{n}$ denotes the $(d-1)$ dimensional part of the closure in $\bar{S}$ of the subset of $S$ formed by the points $p \in S$ where $\sigma(p)$ has exact order $n$. In particular, $X_{n}$ is defined over the number field $k$. By the above remark, the degree of their absolutely irreducible components tend to infinity.

A main point in the proof is the proposition below. In its statement we denote by $\mu$ the normalized invariant measure on the torus

$$
\left(S_{1}\right)^{d-1}=\left\{\left(\theta_{1}, \ldots, \theta_{d-1}\right) \in \mathbb{C}^{d-1}:\left|\theta_{i}\right|=1, \forall i=1, \ldots, d-1\right\}
$$

Proposition 2.7. There exist an index $\alpha$, a compact semi-algebraic set $\Gamma_{\alpha} \subset U_{\alpha}$, positive real numbers $c, \delta$ such that

$$
\mu\left(\left\{\theta \in\left(S_{1}\right)^{d-1}: \text { for infinitely many } n \in \mathcal{N}_{\alpha},\left|\Gamma_{\alpha} \cap \mathcal{C}_{\theta} \cap X_{n}\right|>c \operatorname{deg} X_{n}\right\}\right)>\delta .
$$

The content of the proposition includes the fact that the set in brackets is measurable.

The meaning of the proposition is obtaining a compact set $\Gamma_{\alpha}$ such that, restricing our torsion n hypersurfaces to $\Gamma_{\alpha}$, we obtain suitably many intersection points with each curve $\mathcal{C}_{\theta}$ in a subset of $\theta$ positive measure. This shall be useful.

Recall that the intersection product $\mathcal{C}_{\theta} \cdot X_{n}$ equals the degree of $X_{n}$; however it could a priori happen that the intersection points would excape to infinity for growing $n$, and this would be a serious obstacle in our proof.

The next subsection is devoted to the proof of Proposition 2.7.

\subsection{Uniform distribution, measures}

We use the previous notation: $\lambda: S \rightarrow \mathbb{A}^{d-1}$ is a rational map defined outside a finite base locus, whose fibers are open subsets of the curves $\mathcal{C}_{\theta}$ (namely the fibers are the $\mathcal{C}_{\theta}$ with points at infinity removed). The curves $\mathcal{C}_{\theta}$ satisfy the conclusion of Lemma 2.5.

Recall that for $n \in \mathcal{N}, X_{n}$ is the union of the hypersurfaces of exact torsion order $n$; it is a pure $d-1$ dimensional subvariety of $S$ defined over $k$. 
We fix for the moment an index $n \in \mathcal{N}$. Note that $X_{n} \cap T$ is a fixed codimension two closed subset of $\bar{S}$. Then there exists a proper Zariski closed subset $Z_{n} \subset \mathbb{A}^{d-1}$ such that for each $\theta \in \mathbb{A}^{d-1} \backslash Z_{n}$ :

- $\mathcal{C}_{\theta} \cap X_{n}$ does not intersect infinity (so it is contained in $S$ );

- $\mathcal{C}_{\theta}$ intersects $X_{n}$ at finitely many points and transversally therehin, hence with multiplicity 1.

(For the second condition to hold just include in $Z_{n}$ the ramification divisor of the $\operatorname{map} \lambda: X_{n} \rightarrow \mathbb{P}_{d-1}$.)

As a consequence, for all $\theta$ outside $Z_{n}$, we have

$$
\mid\left(\mathcal{C}_{\theta} \cap X_{n} \cap S \mid \geq \operatorname{deg} X_{n}\right.
$$

(Recall that the degree of a hypersurface in $S$ is equal to its intersection product with any curve $\mathcal{C}_{\theta}$; actually the above inequality is in fact an equality.)

Let now $\mathbf{m}=\left(m_{1}, \ldots, m_{d-1}\right) \in \mathbb{N}^{d-1}$ be a multi-index; at the end of the proof each $m_{i}$ will tend to infinity. The entries $m_{i}$ are chosen in such a way that the Galois group $\operatorname{Gal}\left(k\left(\Omega_{\mathbf{m}}\right) / k\right)$ acts transitively on the set

$$
\Omega_{\mathbf{m}}:=\left\{\left(\theta_{1}, \ldots, \theta_{d-1}\right) \in S_{1}^{d-1}: \theta_{i} \text { has order } m_{i}\right\} .
$$

It suffices that the $m_{i}$ are pairwise coprime and divisible only by primes large enough in term of $k$. We also require that $\Omega_{\mathbf{m}} \cap Z_{n}=\emptyset$, which is ensured from the above assumptions in the $m_{i}$ are sufficiently large.

Let $\theta \in \Omega_{\mathbf{m}}$; by Uniform Silverman's Theorem (see Appendix A), the height of each point in $\mathcal{C}_{\theta} \cap X_{n}$ is bounded (independently of $\mathbf{m}, \theta \in \Omega_{\mathbf{m}}$ and $n$ ) since the value by the section is torsion:

$$
h(p) \leq C_{1}, \quad \forall p \in \mathcal{C}_{\theta} \cap X_{n}
$$

(The height appearing on the left can be taken to be a height function associated to the given embedding of $\bar{S}$, so it is associated to the divisor at infinity of $S$ ).

Let now $f_{1}, \ldots, f_{r}$ be generators of the algebra $k[S]$ (for instance $r$ is the dimension of an affine space containing $S$ and $f_{1}, \ldots, f_{r}$ are the coordinate functions). Then for each $p \in S(\bar{k})$ and each $i=1, \ldots, r$,

$$
h\left(f_{i}(p)\right) \leq C_{2}(h(p)+1) .
$$

Our plan is to define a compact set $\Gamma \subset S$ by a system of inequalities involving the $f_{1}, \ldots, f_{r}$, in such a way that $X_{n} \cap \mathcal{C}_{\theta}$ will have 'many' points in $\Gamma$ for 'many' choices of the parameter $\theta$. 
Recalling that the index $n$ is fixed, let us put for $\theta \in S_{1}^{d-1}$,

$$
I_{\theta}=I_{\theta, n}=\mathcal{C}_{\theta} \cap X_{n}
$$

We also define $G=G(\mathbf{m})=\operatorname{Gal}\left(k\left(\Omega_{\mathbf{m}}\right) / k\right)$ and

$$
U_{\mathbf{m}}=\bigcup_{\theta \in \Omega_{\mathbf{m}}} I_{\theta}
$$

Pick a function $f$ in $\left\{f_{1}, \ldots, f_{r}\right\}$. Then from (2.3), for every $p \in X_{n}(\bar{k})$

$$
C_{2}|G|(h(p)+1) \geq|G|\left(h(f(p)) \geq \sum_{\sigma \in G}\left(\log ^{+}\left|f\left(p^{\sigma}\right)\right|\right),\right.
$$

where the involved absolute value is the usual complex absolute value. The second inequality expresses the fact that the height is at least its archimedean part.

Now, as remarked above, by Uniform Silverman, for every $\theta \in \Omega_{\mathbf{m}}$ and every $p \in$ $X_{n} \cap \mathcal{C}_{\theta}, h(p) \leq C_{1}$ by (2.2). Then, for each real number $H \geq 1$, the number of $\sigma \in G$ such that $\left|f\left(p^{\sigma}\right)\right|>H$ is bounded by $\left(C_{1}+1\right) C_{2}|G| / \log H$. It follows that there exists $H_{0}$ such that

$$
\left|\left\{\sigma \in G:\left|f\left(p^{\sigma}\right)\right| \leq H_{0}\right\}\right| \geq|G|(1-1 / 2 r) .
$$

Since the set $U_{\mathbf{m}}$ is $G$-invariant, we may partition it in $G$-orbits and deduce that for at least $\left|U_{\mathbf{m}}\right| / 2$ elements $p \in U_{\mathbf{m}},\left|f_{i}(p)\right| \leq H_{0}$ for all $i=1, \ldots, r$. In other words, for at least $\left|U_{\mathbf{m}}\right| / 2$ points $p \in U_{\mathbf{m}}$, all the coordinates of $p$ are bounded by $H_{0}$, so $p$ lies in a fixed compact set $\Gamma$ in $S$. Note that this compact set is independent of $n$, because $C_{1}, C_{2}$ are independent of $n$, due to Uniform Silverman.

Observe now that $\left|U_{\mathbf{m}}\right| \geq \operatorname{deg} X_{n} \cdot\left|\Omega_{\mathbf{m}}\right|$ (Here we are using that $\Omega_{\mathbf{m}}$ does not intersect $Z_{n}$ and also that for two distinct $\theta, \theta^{\prime} \in \Omega_{\mathbf{m}}$ the two curves $\mathcal{C}_{\theta}, \mathcal{C}_{\theta^{\prime}}$ intersect only in the (finite) base locus, so in particular outside $X_{n}$ if $n$ is sufficiently large).

Let us put

$$
\gamma(\theta):=\left|I_{\theta} \cap \Gamma\right|=\left|\mathcal{C}_{\theta} \cap X_{n} \cap \Gamma\right|
$$

and note that $\gamma(\theta) \leq \operatorname{deg} X_{n}$.

By the above consideration,

$$
\sum_{\theta \in \Omega_{\mathbf{m}}} \gamma(\theta) \geq \operatorname{deg} X_{n} \cdot \frac{\left|\Omega_{\mathbf{m}}\right|}{2} .
$$

We now use the following lemmas

Lemma 2.8. Let $\mu$ be the invariant measure on the torus $S_{1}^{d-1}$. Let $I \subset S_{1}^{d-1}$ be a product of intervals. Suppose that for an infinite sequence of $\mathbf{m}=\left(m_{1}, \ldots, m_{d-1}\right)$ as above and for a positive real $c$ with $0<c<1 / 2$ we have

$$
\sum_{\theta \in \Omega_{\mathbf{m}} \cap I} \gamma(\theta) \geq c \operatorname{deg} X_{n} \cdot\left|\Omega_{\mathbf{m}} \cap I\right| .
$$


Then we have the lower bound:

$$
\mu\left(\left\{\theta \in I: \gamma(\theta) \geq \frac{c}{2} \cdot \operatorname{deg} X_{n}\right\}\right) \geq \frac{c}{2} \mu(I) .
$$

Proof. We remark that in general for a rational map $\pi: Y \rightarrow Z$ the set of points of $Z$ where the fiber has cardinality $\geq N$ can be described as follows: take the $N$-fold fiber product $Y^{(N)}$ over $Z$, endowed with the natural map $\pi_{N}: Y^{(N)} \rightarrow Z$, and consider the grand diagonal $\Delta_{N}$. The points in questions are those $z \in Z$ in the image $\pi_{N}\left(Y^{(N)} \backslash \Delta_{N}\right)$.

In our case we apply this with $Y=X_{n} \cap \Gamma \cap\left(\bigcup_{\theta \in I} \mathcal{C}_{\theta}\right), Z=I \subset S_{1}^{d-1}$ and $\pi=\lambda$. Since this set $Y$ is semi-algebraic, so are $Y^{(N)} \backslash \Delta_{N}$ and its projection under $\lambda$.

Let us denote by $\Sigma$ the semi-algebraic set

$$
\Sigma=\left\{\theta \in I: \gamma(\theta) \geq \frac{c}{2} \cdot \operatorname{deg} X_{n}\right\} \subset S_{1}^{d-1} .
$$

The boundary of this set is by standard properties of semi-algebraic sets a finite union of analytic subvarieties Lipschitz-parametrizable.

We make a deduction from the inequality in the assumption. Let us put

$$
N_{\mathbf{m}}:=\left|\left\{\theta \in \Omega_{\mathbf{m}} \cap I: \gamma(\theta) \geq \frac{c}{2} \operatorname{deg} X_{n}\right\}\right|=\left|\Sigma \cap \Omega_{\mathbf{m}}\right| .
$$

We recall that in any case $\gamma(\theta) \leq \operatorname{deg} X_{n}$. We clearly have

$$
\sum_{\theta \in \Omega_{\mathbf{m}} \cap I} \gamma(\theta) \leq N_{\mathbf{m}} \operatorname{deg} X_{n}+\frac{c}{2}\left(\left|\Omega_{\mathbf{m}} \cap I\right|-N_{\mathbf{m}}\right) \operatorname{deg} X_{n} .
$$

We deduce from the main assumption of the lemma

$$
N_{\mathbf{m}} \geq \frac{c}{2}\left|\Omega_{\mathbf{m}} \cap I\right|\left(1-\frac{c}{2}\right)^{-1} \geq \frac{c}{2}\left|\Omega_{\mathbf{m}} \cap I\right|
$$

Reading the angles of the $\theta \in S_{1}^{d-1}$ in terms of rational points in $[0,1]^{d-1}$, we have reached the conclusion that the number of rational points of the shape $\left(a_{1} / m_{1}, \ldots, a_{d-1} / m_{d-1}\right) \in$ $\mathbb{Q}^{d-1} \cap[0,1]^{d-1}$, for $\left(m_{1}, \ldots, m_{d-1}\right)=\mathbf{m}$ counted in $N_{\mathbf{m}}$, is at least $\frac{c}{2}\left|\Omega_{\mathbf{m}} \cap I\right|$. In turn, by standard (and easy) uniform distribution results on the residue classes coprime with the modulus, we have that $\left|\Omega_{\mathbf{m}} \cap I\right| \sim\left|\Omega_{\mathbf{m}}\right| \cdot \mu(I)$. On the other hand, by the mentioned results on its boundary, the set $\Sigma$ contains asymptotically $\left|\Omega_{\mathbf{m}} \cap I\right|$ rational points of that type, and the result follows.

Lemma 2.9. Let $(I, \mu)$ be a measure space with finite total measure, $\varphi_{n}: I \rightarrow[0,1]$ be an infinite sequence of measurable functions with $0 \leq \varphi_{n} \leq 1, c_{1}, c_{2}$ positive real numbers. Suppose that for all $n$

$$
\mu\left(\left\{\theta \in I: \varphi_{n}(\theta) \geq c_{1}\right\}\right) \geq c_{2} \mu(I) .
$$

Then the set of $\theta \in I$ such that $\varphi_{n}(\theta) \geq c_{1}$ for infinitely many $n$ has measure $\geq c_{2} \mu(I)$. 
In fact this lemma could be stated for an arbitrary sequence of measurable subsets, not necessarily in terms of functions.

Proof. We first note that the set in question is measurable, since its complement consists of the set of $\theta$ such that $\varphi_{n}(\theta) \geq c_{1}$ for only finitely many $n$; so the complement is the union over the finite subsets $K$ of $\mathbb{N}$ of the intersections $\cap_{n \in K}\left\{\theta: \varphi_{n}(\theta) \geq c_{1}\right\} \cap_{n \notin K}$ $\left\{\theta: \varphi(\theta)<c_{1}\right\}$, hence it is measurable.

Now, since for all $n \in \mathbb{N}, \int_{I} \varphi_{n} d \mu \geq c_{1} c_{2} \mu(I)$, we have for all $N \in \mathbb{N}$

$$
\int_{I}\left(\sum_{n \leq N} \varphi_{n}\right) d \mu \geq c_{1} c_{2} \mu(I) N .
$$

Let us denote by $K(\theta, N)$ the integer

$$
K(\theta, N)=\left|\left\{n \leq N: \varphi_{n}(\theta) \geq c_{1}\right\}\right| .
$$

Then, setting $\mu_{i}=\mu\left(\left\{\theta: \varphi_{i}(\theta) \geq c_{1}\right\}\right)$, we have

$$
\int_{I} K(\theta, N) d \mu \geq \mu_{1}+\ldots+\mu_{N}
$$

By our assumption, $\mu_{i} \geq c_{2} \mu(I)$ for all $i$. Then

$$
\int_{I} K(\theta, N) d \mu \geq N c_{2} \mu(I)
$$

Let $k(\theta)=\limsup _{N \rightarrow \infty} K(\theta, N)=\sup _{N} K(\theta, N)$. Let $I=\Omega_{\infty} \cup \Omega_{0} \cup \Omega_{1} \cup \ldots$ be the partition of $I$ defined by

$$
\Omega_{j}=\{\theta: k(\theta)=j\} .
$$

It is easily seen as above that all the $\Omega_{j}$ are measurable. Then $\mu(I)=\mu\left(\Omega_{\infty}\right)+\mu\left(\Omega_{0}\right)+$ $\mu\left(\Omega_{1}\right)+\ldots=m_{\infty}+m_{0}+m_{1}+\ldots$, for $m_{i}:=\mu\left(\Omega_{i}\right)$. We want to prove that $m_{\infty} \geq c_{2} \mu(I)$. Note that the tails $\sigma_{i}:=m_{i}+m_{i+1}+\ldots$ of the converging sum $m_{0}+m_{1}+m_{2}+\ldots$ tend to zero for $i \rightarrow \infty$. We have

$$
\begin{array}{ccc}
\int_{I} K(\theta, N) d \mu & \leq & \int_{I} \min (N, k(\theta)) d \mu \\
& = & m_{1}+\ldots+(N-1) m_{N-1}+N\left(m_{\infty}+m_{N}+m_{N+1}+\ldots\right) \\
& = & \left(\sum_{j=1}^{N-1} j m_{j}\right)+N \sigma_{N}+N m_{\infty}
\end{array}
$$

We note that $\sum_{j=1}^{N-1} j m_{j}=\sigma_{1}+\ldots+\sigma_{N-1}$. Since $\sum_{j \geq 1} m_{j}$ converges, we have $\sigma_{N} \rightarrow 0$ for $N \rightarrow \infty$; this implies in particular that $\sigma_{1}+\ldots+\sigma_{N-1}=o(N)$.

Hence $\int_{I} K(\theta, N) d \mu=N m_{\infty}+o(N)$, and the result follows from the lower bound $(2.5)$.

Now Proposition 2.7 follows from (2.4) using the above lemmas (we apply the second lemma with $\left.\varphi_{n}(\theta)=\frac{\gamma(\theta)}{\operatorname{deg} X_{n}}\right)$. The fact that the compact set can be taken inside $U_{\alpha}$ follows from the finiteness of the cover $\left\{U_{\alpha}\right\}_{\alpha}$. 


\subsection{Proof of Theorem 2.1}

We argue by induction on the dimension $d$ of the base $S$. The case $d=1$ is contained in [16]. We then assume to have proved our assertion for all bases of dimension $<d$, and proceed to deduce the theorem in dimension $d$.

This induction hypothesis also allows us to assume that certain 'generic' properties of $\pi$ hold everywhere on $S$ (for we may restrict to the Zariski open dense subset where they hold, and then use induction on the complement). For instance, we can use this remark in Appendix A.

Proof. Fix an index $\alpha$ as in Proposition 2.7. We can suppose that $\Gamma_{\alpha}$ is semialgebraic and the closure of an open subset of $S$. The image $\mathfrak{b}\left(\Gamma_{\alpha}\right)$ is a compact subanalytic subset $B \subset \mathbb{R}^{4}$. For each positive integer $n \in \mathbb{N}$ the set $\mathfrak{b}\left(X_{n} \cap \Gamma_{\alpha}\right)$ consists of finitely many rational points in $B \cap \mathbb{Q}^{4}$ : in fact $\mathfrak{b}$ is locally constant on $X_{n}$ and $X_{n} \cap \Gamma_{\alpha}$ has only finitely many connected component, since it is semi-algebraic. The denominators of these rational numbers divide $n$; by compacity, their numerators are also bounded by $O(n)$.

By a very weak form of Proposition 2.7, there exists a $\theta$ such that the set $X_{n} \cap \Gamma_{\alpha} \cap \mathcal{C}_{\theta}$ has $\geq c \operatorname{deg} X_{n}$ points for all $n$ in an infinite set $\mathcal{N}_{\theta}$ and a fixed number $c>0$. (Actually Proposition 2.7 provides a set of such $\theta$ of positive measure.)

Suppose first that $\left|\mathfrak{b}\left(X_{n} \cap \Gamma_{\alpha}\right)\right| / \operatorname{deg} X_{n} \rightarrow 0$ for $n \in \mathcal{N}_{\theta}$. Then, for every $N$ there exist infinitely many integers $n \in \mathcal{N}_{\theta}$ and points $\xi_{n} \in B \cap \mathbb{Q}^{4}$ such that $\mathfrak{b}$ takes the value $\xi_{n}$ more than $N$ times on $X_{n} \cap \Gamma_{\alpha} \cap \mathcal{C}_{\theta}$ (here we use the fact that $\operatorname{deg} X_{n}$ tends to infinity). This implies that the map $\mathfrak{b}: \Gamma_{\alpha} \cap \mathcal{C}_{\theta} \rightarrow \mathbb{R}^{4}$ has a fiber above the point $\xi_{n}$ of cardinality exceeding $N$. However, by Gabrielov theorem the number of connected components of the fibers of this map is uniformly bounded (see Theorem 3.14 of [5]). Hence we deduce that if $N$ is large enough the said fiber must have a component of positive dimension. In turn, this would imply by analytic continuation that the whole $\mathcal{C}_{\theta}$ is a torsion curve. However this is impossible because $\mathcal{C}_{\theta}$ intersects infinitely many $X_{n}$ in $\Gamma_{\alpha}$ (or else it would follow also from the fact that $\mathcal{C}_{\theta}$ contains a point of good reduction).

We now consider the case when the map $\mathfrak{b}$ takes more than $\epsilon \operatorname{deg}\left(X_{n}\right)$ values on $X_{n}$ for some fixed $\epsilon>0$ and infinitely many $n \in \mathcal{N}_{\theta}$. We shall obtain a contradiction by using the following results:

Proposition 2.10. There exists a positive constant c $>0$ such that the degrees $\operatorname{deg} X_{n}$ of the torsion hypersurfaces satisfy

$$
n^{c} \ll \operatorname{deg}\left(X_{n}\right) \ll n^{2}
$$


Proof. We first prove the upper bound $\operatorname{deg} X_{n} \ll n^{2}$.

Let $S_{n}$ be the set of $p$ in $S$ with $n \sigma(p)=0$, a quasi-projective subset. Because $S$ is irreducible and $\sigma$ is not torsion, we have $\operatorname{dim} S_{n} \leq d-1$. As the Zariski closure $\overline{X_{n}}$ also of dimension $d-1$ lies in $\overline{S_{n}}$, it must be a union of components of $\overline{S_{n}}$ (and $\operatorname{dim} S_{n}=d-1$ ). Now $\overline{S_{n}}$ is defined in $\bar{S}$ by the equations expressing multiplication by $n$ evaluated at $\sigma(p)$. According to Serre [20] (and viewing the generic fiber of our abelian scheme as an abelian variety over a function field), we can choose a quasi-projective embedding of $\mathcal{A}$ such that the multiplication equations have degrees $n^{2}$ and are everywhere defined. Thus $\overline{S_{n}}$ is defined in $\bar{S}$ by equations of degrees $\ll n^{2}$. Thus standard results show that the sum of the degrees of the $(d-1)$-dimensional components of $\overline{S_{n}}$ is $\ll n^{2}$. So the same bound holds for $\operatorname{deg} \overline{X_{n}}$.

We now prove the lower bound with any $c<1 / 513$.

Suppose that for some $c>0$ there are infinitely many torsion hypersurfaces $X_{n}$ with $\operatorname{deg} X_{n}<n^{c}$.

Let us consider a generic line $r \subset \mathbb{A}^{d-1}$, defined over $k$, in the space $\mathbb{A}^{d-1}$; the preimage $\lambda^{-1}(r)$ is a surface $S^{\prime} \subset S$. By generic for a line $r$ we mean that for two generic distinct points $\left(\theta_{1}, \ldots, \theta_{d-1}\right)$ and $\left(\theta_{1}^{\prime}, \ldots, \theta_{d-1}^{\prime}\right)$ on $r$, for each $i=1, \ldots, d-1$ we shall have $\theta_{i} \neq \theta_{i}^{\prime}$.

We shall work over this surface $S^{\prime}$, fibered over $r$, which we can suppose to be nontorsion for $\sigma$ and such that $\mathcal{A}_{s}$ is generically simple for $s \in S^{\prime}$. Our aim is finding, for each sufficiently large value of $n$, a curve $\mathcal{C}_{\theta}$ in $S^{\prime}$ (for some $\theta \in r$ ) intersecting $X_{n}$ at an algebraic point $p \in S^{\prime}$, i.e. outside infinity. Then we shall apply David's estimate to the variety $\mathcal{A}_{p}$ and the point $\sigma(p)$.

Observe that $X_{n}$ intersects infinity at $\leq C_{1} \operatorname{deg} X_{n}$ points (here and in the sequel $C_{1}, C_{2} \ldots$ will denote numbers depending on all the data $\mathcal{A} \rightarrow S, \sigma, r$ but not on $n$ ). Since the line $r$ is generic in the sense just mentioned, by our construction (see Lemma 2.5, part (4)) no two distinct curves $\mathcal{C}_{\theta}$ (for distinct choices of $\theta \in r$ ) can meet at infinity. Hence, if we consider a sub-set of $r$ consisting of more than $C_{1} \operatorname{deg} X_{n}$ elements, for at least one $\theta$ in this subset the curve $\mathcal{C}_{\theta}$ will intersect $X_{n}$ inside $S^{\prime}$ (i.e. outside infinity). We shall choose this finite subset of $r$ as the set of $k$-rational points of height $\leq B$ for some $B=B(n)$.

For our purpose we define, for each $n \geq 1$,

$$
B=B(n):=n^{b}
$$

for some suitable $b>0$; it will turn out that $b=1 / 513$ will be a convenient choice, but many other choices for the function $B(n)$ are possible, as it will be clear from the sequel.

Note that for large $n$ there are $\geq \exp (B)=\exp \left(n^{b}\right)$ points $\theta \in r$ of (logarithmic) height $h(\theta) \leq B$ (the estimate holds for $n$ large enough with respect to the height of the 
line $r$ ). So for large $n$ we can find a point $\theta \in r$ with $h(\theta) \leq n^{b}$ such that $\mathcal{C}_{\theta}$ intersects $X_{n}$ in $S^{\prime}$.

By the Uniform Silverman's Theorem, for each $\theta \in r$ of height $\leq B$ such that $\mathcal{C}_{\theta}$ is non-torsion, the points $p \in \mathcal{C}_{\theta}$ for which $\sigma(p)$ is torsion satisfy $h(p) \leq C_{2} B$. Those points which lie on $X_{n}$ (and so are of exact order $n$ ) satisfy also $[k(p): k] \leq \operatorname{deg} X_{n} \operatorname{deg} \mathcal{C}_{\theta}=C_{3} n^{c}$. Let choose one such point $p$.

The abelian variety $\mathcal{A}_{p}$ will be defined over the number field $\kappa:=k(p)$ and satisfies

$$
h\left(\mathcal{A}_{p}\right) \leq C_{4} n^{b}, \quad[\kappa: \mathbb{Q}] \leq C_{5} n^{c} .
$$

We apply the estimates in Proposition 2.3 to the torsion point $P:=\sigma(p) \in \mathcal{A}_{p}(\kappa)$. Now for $g=2$ we have $G=256$. Thus Proposition 2.3 gives

$$
n \ll\left(C_{6} n^{c} \cdot n^{b}\right)^{256} .
$$

This gives the required contradiction with $c=b=1 / 513$.

The next proposition is a theorem of Pila [17].

Proposition 2.11. Let $B \subset \mathbb{R}^{n}$ be a compact sub-analytic subvariety. Suppose that the set of rational points $B \cap \mathbb{Q}^{n}$ of $B$ satisfies the growth condition:

$$
\left|\left\{\xi \in \mathbb{Q}^{n} \cap B: H(\xi) \leq N\right\}\right| \geq N^{c}
$$

for infinitely many $N$ and a fixed $c>0$. Then the set $B$ contains a semi-algebraic subvariety of dimension 1.

The next result can be deduced from the already mentioned theorem of André on algebraic independence of abelian integrals, given as Theorem 2.4:

Proposition 2.12. Under our assumptions, the set $B=\mathfrak{b}\left(\mathcal{C}_{\theta} \cap \Gamma_{\alpha}\right)$ does not contain algebraic arcs.

Proof. Let $\gamma \subset \mathfrak{b}\left(\Gamma_{\alpha} \cap \mathcal{C}_{\theta}\right)$ be an algebraic arc and consider the real analytic set $\mathfrak{b}^{-1}(\gamma)=V \subset \Gamma_{\alpha}$. Let $r$ be the transcendence degree of the eight complex-valued functions $f_{1}, \ldots, f_{8}$ arising as coordinates of the periods, restricted to $V$; we have $r \leq 8$ and actually the period functions can be algebraicailly dependent even on the whole $S$ (e.g. in presence of a constant part for the family). The components $b_{1, \alpha}, \ldots, b_{4, \alpha}$ of the Betti map, when restricted to $V$, have transcendence degree at most 1 , in the sense any two of them verify a fixed algebraic relation with constant coefficients. So, the transcendence degree of the field generated by $f_{1}, \ldots, f_{8}, b_{1}, \ldots, b_{4}$ is $\leq r+1$; this field contains the two (complex) coordinates of our abelian logarithms restricted to $V \subset \mathcal{C}_{\theta} \cap \Gamma_{\alpha}$. Therefore these two coordinates, restricted to $V$, would be algebraically dependent over $\mathbb{C}\left(f_{1}, \ldots, f_{8}\right)$ when 
restricted to $V$. Since however they are analytic functions on $\mathcal{C}_{\theta} \cap \Gamma_{\alpha}$ and $V$ has real dimension $\geq 1$, they must be algebraically dependent over $\mathbb{C}\left(f_{1}, \ldots, f_{8}\right)$ also on $\mathcal{C}_{\theta}$; but then by Theorem 2.4 applied to $\mathcal{C}_{\theta}$ (recall that by construction the abelian family over $\mathcal{C}_{\theta}$ remains simple and non-isoconstant) the section would be torsion on $\mathcal{C}_{\theta}$, which is excluded by the fact that $\mathcal{C}_{\theta}$ intersects infinitely many $X_{n} .{ }^{4}$

We can now conclude: we are supposing that for infinitely many $n, \mathfrak{b}\left(\Gamma_{\alpha} \cap X_{n}\right)$ contains $\gg \epsilon \operatorname{deg} X_{n}$ points (actually $\mathfrak{b}\left(\Gamma_{\alpha} \cap X_{n} \cap \mathcal{C}_{\theta}\right)$ ), which must be rational of exponential height $\ll n$. By inequality 2.6 of Proposition 2.5 this implies that the set $B \cap \mathbb{Q}^{4}$ contains $\gg n^{c}$ rational points of height $\gg n$; then by Proposition 2.11 such a set $B$ must contain an algebraic arc, which contradicts Proposition 2.12.

REMARK 2.13. One could replace Theorem 2.11 by a stronger version which is uniform in $\theta$. Namely, given a compact region $\Gamma_{\alpha}$, there is an upper bound for the number of rational point of height $\leq n$ in $\mathcal{C}_{\theta} \cap \Gamma_{\alpha}$ valid for every $\theta$, of the form $n^{\epsilon}$ as in Theorem 2.11. This would allow to avoid the full force of Proposition 2.7 (achieved via the measuretheoretic lemma). It would suffice to find some $\theta$ as above intersecting some $X_{n}$ in more then $c \operatorname{deg} X_{n}$ points.

REMARK 2.14. . In the particular case where the rank of the differential of the Betti map is 2 , a simpler path can be followed. The main point is that $\mathfrak{b}$ restricted to a nontorsion curve (e.g. $\mathcal{C}_{\theta}$ ) takes essentially the same values as it takes in the whole $\Gamma_{\alpha}$. Then one can choose an algebraic value of $\theta$ to reduce to the case of curves (over number fields) already treated in [16].

\subsection{Maximal rank of the Betti map}

In this section we give an alternative proof of Theorem 0.1 under the assumption that the generic rank of $\mathfrak{b}$ is 4 . As mentioned above, this argument probably adapts generally and has the advantage of reducing to the algebraic case without appealing to the deep results used in the previous section.

We choose again an index $\alpha$ such that the conclusion of Proposition 2.7 holds.

\footnotetext{
${ }^{4}$ We thank a referee for pointing out that in order to apply Theorem 2.4 we need that the the scheme remains non iso-constant over $\mathcal{C}_{\theta}$. The referee also suggests a possible alternative method, that is to use a theorem of $\mathrm{Ax}$ in addition to André's theorem. See Theorem 4.3 in [4] for a combination of the two theorems.
} 
We denote by $A \subset \Gamma_{\alpha}$ the subset of $\Gamma_{\alpha}$ formed by the points $s$ such that the rank of $d \mathfrak{b}$ in $s$ is $\leq 2$ (recall that by Proposition 1.1 the rank cannot be odd). Note that $A$ is a semi-analytic set of real dimension $\leq 2 d-1$, by our present assumption on the rank, and contains all the hypersurfaces $X_{n}$ (or better all their intersections with $\Gamma_{\alpha}$ ). Let $A^{\prime}$ be the smooth part of $A$; $A^{\prime}$ is obtained on removing finitely many smooth subvarieties of dimension $\leq 2 d-2$ from $A$, we obtain a smooth subvariety $A^{\prime}$ of real dimension $2 d-1$ which still contains all but finitely many $X_{n}$ (otherwise all the hypersurfaces $X_{n}$ would be contained in finitely many connected analytic hypersurfaces, and we would have only finitely many $X_{n}$ as wanted). Moreover, at each point $p \in X_{n} \cap A^{\prime}$ the rank of the restriction of $d \mathfrak{b}$ to $A^{\prime}$ is $\leq 1$, since $\mathfrak{b}$ is constant on the real hypersurface $X_{n} \cap A^{\prime}$ of $A^{\prime}$ passing through $p$. Then, letting $A^{\prime \prime}$ be the following subset of $A^{\prime}$ :

$$
A^{\prime \prime}=\left\{p \in A^{\prime}: \operatorname{rank}_{p}\left(d \mathfrak{b}_{\mid A^{\prime}}\right) \leq 1\right\}
$$

we obtain that $A^{\prime \prime}$ contains open subsets of each but finitely many $X_{n}$ intersecting $\Gamma_{\alpha}$. We also have that $\mathfrak{b}\left(A^{\prime \prime} \cap \Gamma_{\alpha}\right)$ is a sub-analytic subset of $\mathbb{R}^{4}$, of dimension 1 (for if the dimension would be zero, we would have only finitely many $X_{n}$ intersecting $A^{\prime \prime}$ ). By [5], Thm. 6.1(1), it is also semi-analytic, i.e. finite union of analytic arcs and isolated points.

Put $\Gamma_{\alpha}^{\prime \prime}=\Gamma_{\alpha} \cap A^{\prime \prime}$. We know that the set $\cup_{n \geq 1} \mathfrak{b}\left(X_{n} \cap \Gamma^{\prime \prime}\right)$ is an infinite set of rational points in $\mathfrak{b}\left(\Gamma_{\alpha}^{\prime \prime}\right)$. So there is an $\operatorname{arc} B$ in $\mathfrak{b}\left(\Gamma_{\alpha}^{\prime \prime}\right)$ containing infinitely many of these points (since there are only finitely many arcs). We can also suppose that $\mathfrak{b}^{-1}(B)$ contains an open subset of $A^{\prime \prime}$ and that Proposition 2.7 still holds restricting to this set: in other words, the set of $\theta \in\left(S_{1}\right)^{d-1}$ such that $C_{\theta}$ intersects infinitely many $X_{n}$ in $\mathfrak{b}^{-1}(B) \cap A^{\prime \prime}$ has positive measure.

Let us denote by $C$ the intersection of $\mathfrak{b}^{-1}(B) \cap A^{\prime \prime}$ with the set of points where the rational functions $\theta_{1}, \ldots, \theta_{d-1}$ take values of modulus 1 .

The image $\mathfrak{b}(C)$ is a smooth arc in $\mathbb{R}^{4}$ containing infinitely many rational points and morevoer there is a set $\Theta \subset S_{1}^{d-1}$ of positive measure such that $\mathfrak{b}\left(C \cap C_{\theta}\right)$ is infinite for $\theta \in \Theta$. We now distinguish two cases:

First case. There exist only finitely many accumulation points for the set $R:=$ $\cup_{n \geq 1}\left(\mathfrak{b}\left(C \cap X_{n}\right)\right)$.

Recall that for $\theta \in\left(S_{1}\right)^{d-1}$ of positive measure the curve $\mathcal{C}_{\theta}$ intersects infinitely many $X_{n}$ in $C$, so its image by $\mathfrak{b}$ contains infinitely many rational points; since $\mathfrak{b}\left(\mathcal{C}_{\theta} \cap C\right)$ has only finitely many components, it must contain all but finitely many rational points in $B$.

By Gabrielov's Theorem, the set $\mathfrak{b}^{-1}(\xi) \cap C$ has a uniformly bounded number of components, say at most $N$ components, independently of $\xi$. So in particular the first component of the $\lambda$ function on $\left(\mathfrak{b}^{-1}(\xi) \cap C\right)$ is a union of $\leq N$ intervals in $S_{1}$.

Note that by Fubini's theorem the set of $\theta_{1} \in S_{1}$ such that $Z_{\theta_{1}}$ intersects infinitely many $X_{n}$ in $\Gamma_{\alpha}^{\prime \prime}$ has positive measure, so in particular is infinite. Using the finiteness of 
the accumulation points of $R$, we can find $N+2$ points $\theta_{1}^{(1)}, \ldots, \theta_{1}^{N+2}$ such that the sets $\mathfrak{b}\left(Z_{\theta_{1}^{(i)}} \cap \Gamma_{\alpha}^{\prime \prime}\right)$ all contain a single interval $J$ containing an accumulation point for $R$.

Using real coordinates for the circle $S_{1}$ (identified to the interval $[0,1)$ ) we can order these $\theta$ such that $\theta_{1}^{(1)}<\theta_{1}^{(2)}<\ldots<\theta_{1}^{(N+2)}$. Let now $\xi_{1}, \ldots, \xi_{N+1}$ be algebraic points in $S_{1}$ such that

$$
\theta_{1}^{(1)}<\xi_{1}<\theta_{1}^{(2)}<\ldots<\xi_{N+1}<\theta_{1}^{(N+2)} .
$$

By the inductive assumption, the $Z_{\xi_{1}}, \ldots, Z_{\xi_{N+1}}$ intersect only finitely many $X_{n}$, so they give rise to only finitely many rational points of the set $R$. Then there exists a point $\eta \in J$ of the form $\eta=\mathfrak{b}(p)$, for some $p \in X_{n} \cap \Gamma_{\alpha}^{\prime \prime}$, such that $\eta \in \mathfrak{b}\left(Z_{\theta_{1}^{(i)}}\right)$ for all $i=1, \ldots, N+2$, but $\eta \notin \mathfrak{b}\left(Z_{\xi_{i}}\right)$ for all $i=1, \ldots, N+1$. This implies that $\theta_{1}\left(\mathfrak{b}^{-1}(\xi) \cap \Gamma_{\alpha}^{\prime \prime}\right)$ consists of at least $N+1$ disjoint intervals.

Second case: The set $\cup_{n \in \mathbb{N}}\left(\mathfrak{b}\left(C \cap X_{n}\right)\right)$ has infinitely many accumulation points.

Since the image of the points where $\mathfrak{b}_{\mid A^{\prime}}$ has rank 0 is finite, there must be a point $p \in A^{\prime \prime}$ where the differential of $\mathfrak{b}$ restricted to $A^{\prime \prime}$ has rank $\geq 1$ (hence $=1$ ) such that $\mathfrak{b}(p)$ is an accumulation point for $R$. Also, by the same reason, we can and shall suppose that $\mathfrak{b}(p)$ lies in a single arc of $\mathfrak{b}\left(A^{\prime \prime}\right)$ and is an interior point of this arc.

Now, up to analytically changing coordinates in $\mathbb{R}^{4}$, we can suppose that for a neighborhood $D$ of $p$ in $A^{\prime \prime}, \mathfrak{b}(D) \subset \mathbb{R}^{4}$ is the set $(x, 0,0,0)$ for $|x|<\epsilon$. So writing $\mathfrak{b}(s)=$ $\left(x_{1}(s), x_{2}(s), x_{3}(s), x_{4}(s)\right)$, the restriction to $D$ of the function $x_{1}$ has non-zero differential in $p$ and the other $x_{i}$ vanish identically on $D$. Identifying a neighborhood of $p$ in $S$ with an open subset of $\mathbb{R}^{2 d}$, we can speak of the gradient of $x_{1}$ at $p$. This gradient is not perpendicular to the tangent space at $D$ in $p$. Hence we can find real analytic coordinates $\left(t_{1}, \ldots, t_{2 d}\right)$ in a neighborhood of $p$ in $S$ such that: (1) the hypersurface $A^{\prime \prime}$ is defined by the equation $t_{1}=0$ and (ii) $\frac{\partial}{\partial t_{1}}\left(x_{1}\right) \neq 0$. The last condition is equivalent to the independence of the differentials of $x_{1}$ and $t_{1}$ at the point $p$, hence in a neighborhood of the point $p$ in $S$.

Let us choose regular functions $f_{1}, \ldots, f_{d-1} \in \overline{\mathbb{Q}}[S]$ such that their differentials in $p$, together with the differentials of $x_{1}$, and $t_{1}$, generate the cotangent space in $p$. By this we mean that, viewing $f_{1}, \ldots, f_{d-1}$ as maps to $\mathbb{R}^{2}$, the corresponding map $\left(f_{1}, \ldots, f_{d-1}, x_{1}, t_{1}\right)$ to $\mathbb{R}^{2 d}$ has maximal rank in $p$.

This map is then surjective to an open subset of $\mathbb{C}^{d-1} \times \mathbb{R} \times \mathbb{R}$, containing the point $\left(f_{1}(p), \ldots, f_{d-1}(p), 0,0\right)$. We can then find an algebraic point $\xi \in \overline{\mathbb{Q}}^{d-1}$ such that: (i) $\left(f_{1}, \ldots, f_{d-1}\right)^{-1}(\xi)$ is an algebraic curve $\mathcal{C}$ containing a point above which the abelian variety is simple (use Masser's Theorem 2.2); (ii) the image of the map $\left(x_{1}, t_{1}\right): \mathcal{C} \rightarrow \mathbb{R}^{2}$ contains a neighborhood of the image of $p$ by $\left(x_{1}, t_{1}\right)$. In particular, restricting $x_{1}$ to $\mathcal{C} \cap A^{\prime \prime}=\mathcal{C} \cap t_{1}^{-1}(0)$ we obtain that $x_{1}$ takes all the values close to the values taken at $p$; but then $\mathfrak{b}$ takes in $\mathcal{C} \cap A^{\prime \prime}$ all the values in a neighborhood of $\mathfrak{b}(p)$ in $\mathfrak{b}\left(A^{\prime \prime}\right)$. Then we 
apply [13] to conclude.

\section{Proof of Theorem 0.1}

In this short paragraph we just deduce Theorem 0.1, which holds for schemes defined over the field of complex numbers, from Theorem 2.1, where the ground field was supposed to be a number field.

We first recall that Theorem 0.1 was already proved in [16] for non-simple abelian schemes of relative dimension two, so we suppose the scheme $\mathcal{A} \rightarrow S$ appearing in Theorem 0.1 is simple. A field of definition for $\mathcal{A}, S$, the map $\mathcal{A} \rightarrow S$ and its section $\sigma: S \rightarrow \mathcal{A}$ can be taken to be a subfield $K \subset \mathbb{C}$, finitely generated field over $\mathbb{Q}$. Then $K$ can be viewed as the function field of an affine variety $B$ over a number field $k$ and $K(S)$ as a function field of a variety $S^{\prime}$, endowed with a (rational) projection $S^{\prime} \rightarrow B$, defined over $k$, whose generic fiber is an open subset of $S$. Let $d=\operatorname{Tr} \operatorname{deg}(K)+\operatorname{dim} S=\operatorname{dim} B+\operatorname{dim} S$ be the dimension of $S^{\prime}$. Up to eliminating possibly a closed proper subset of $S^{\prime}$, we obtain a well defined projection $S^{\prime} \rightarrow B$; the rational points in $S(K)$ correspond to rational sections $B \rightarrow S^{\prime}$ defined over $k$. Also, from the variety $\mathcal{A}$ one can obtain a variety $\mathcal{A}^{\prime}$ defined over $k$, endowed with a rational map $\mathcal{A}^{\prime} \rightarrow S^{\prime}$; to a section $\sigma: S \rightarrow \mathcal{A}$ defined over $K$ corresponds a rational section $\sigma^{\prime}: S^{\prime} \rightarrow \mathcal{A}^{\prime}$ defined over $k$.

Now, every hypersurface in $S$ defined over $K$ gives rise to a hypersurface in $S^{\prime}$ defined over $k .{ }^{5}$. Since the torsion hypersurfaces relative to $\sigma$ are all defined over $K$, they give rise to torsion hypersurfaces of $S^{\prime}$ relative to $\sigma^{\prime}$, defined over $k$. The finiteness Theorem 2.1 proved in last section then implies Theorem 0.1 .

\section{APPENDIX A: Uniform Silverman's Theorem}

Let $S$ be a smooth affine algebraic variety of dimension $d$ over $\overline{\mathbb{Q}}, \varphi: S \rightarrow \mathbb{P}_{d-1}$ a morphism whose generic fibers $\varphi^{-1}(\lambda)=: \mathcal{C}_{\lambda}$ are geometrically irreducible.

As a rule, we shall denote by $\lambda$ a $\overline{\mathbb{Q}}$-generic point of $\mathbb{P}_{d-1}$, while algebraic specializations of $\lambda$ shall be denoted by the letter $\theta$.

Theorem 4.1. Let $\pi: \mathcal{A} \rightarrow S$ be a family of abelian varieties over $S$, defined over a number field. Let $\sigma: S \rightarrow \mathcal{A}$ be a non-torsion section. Let $\varphi: S \rightarrow \mathbb{P}_{d-1}$ be a morphism as before. Then, there exist an open dense subset $S_{0} \subset S$, a positive real number $C_{1}>0$ depending only on $\sigma$ such that for all $\theta \in \mathbb{P}_{d-1}(\overline{\mathbb{Q}})$, with the property that the restriction

\footnotetext{
${ }^{5}$ note that the vice versa does not necessarily hold
} 
of $\sigma$ to $\mathcal{C}_{\theta}$ is non-torsion, every point $p \in \mathcal{C}_{\theta} \cap S_{0}(\overline{\mathbb{Q}})$ such that $\sigma(p)$ is torsion satisfies

$$
h(p)<C_{1}(h(\theta)+1) .
$$

Remarks

-Here the height of $p$ is defined by any previously fixed projective embedding of $S$.

-If the point $\theta$ is fixed, this is a corollary of Silverman's specialization bound applied to the family $\mathcal{A} \rightarrow \mathcal{C}_{\theta}$.

Proof: We use the setting of Chapter 12 in Lang's book [9]. We note at once that by induction on $\operatorname{dim} S$ we may replace in the sequel $S$ with a Zariski open dense subset.

We can compactify the scheme $\mathcal{A} \rightarrow S$ above a suitable dense open subset $S_{0}$ of $S$, to obtain a morphism $\bar{\pi}: \overline{\mathcal{A}} \rightarrow \bar{S}$ between projective varieties which coincides with our previous abelian scheme above $S_{0}$ and is flat above $S_{0}$.

Take an ample divisor on $\overline{\mathcal{A}}$, inducing an ample symmetric divisor on each abelian variety $\mathcal{A}_{s}:=\pi^{-1}(s)$, for $s$ in $S_{0}$. This divisor gives an embedding $\mathcal{A} \hookrightarrow \mathbb{P}_{N}$, hence a naive height in $\mathcal{A}(\overline{\mathbb{Q}})$. For each $s \in S_{0}$, we also get en embedding of the abelian variety $\mathcal{A}_{s}$ into $\mathbb{P}_{N}$, so a (naive) height in $\pi^{-1}(s)$ induced by that of $\mathcal{A}$.

Let now $\sigma: S_{0} \rightarrow \mathcal{A}$ be a non-torsion section. Composing with the embedding $\mathcal{A} \hookrightarrow \mathbb{P}_{N}$ we obtain a map $S_{0} \rightarrow \mathbb{P}_{N}$. Recall the construction of a rational map $\lambda: S \rightarrow \mathbb{P}_{d-1}$; for the generic $\lambda \in \mathbb{P}_{d-1}$, we can consider the restriction of the section $\sigma$ to the curve $\mathcal{C}_{\lambda}$, which will be denoted by $\sigma_{\lambda}$. Then we can write $\sigma_{\lambda}: \mathcal{C}_{\lambda} \rightarrow \mathcal{A} \hookrightarrow \mathbb{P}_{N}$ as

$$
\left(f_{0, \lambda}: \ldots: f_{N, \lambda}\right): \mathcal{C}_{\lambda} \rightarrow \mathbb{P}_{N}
$$

More generally, replacing $\sigma$ by $M \cdot \sigma$, we obtain corresponding maps $\left(f_{0, \lambda}^{(M)}: \ldots\right.$ : $\left.f_{N, \lambda}^{(M)}\right): \mathcal{C}_{\lambda} \rightarrow \mathbb{P}_{N}$. Here each $f_{i, \lambda}^{(M)}$ is a rational function on the curve $\mathcal{C}_{\lambda}$. We may also suppose $f_{0, \lambda}^{(M)}=1$.

Provided $\theta$ is an algebraic point in $\mathbb{P}_{d-1}$ lying outside a proper Zariski closed set, we may specialize at $\lambda=\theta$ obtaining corresponding functions $f_{i, \theta}^{(M)}$ on the irreducible curve $\mathcal{C}_{\theta}$ (which are irreducible by Lemma $2.5(1)$ ).

Remark. In the proof of the Theorem and of the subsequent lemmas, we shall choose a fixed $M$; hence we can argue for specializations $\theta$ of $\lambda$ outside some prescribed hypersurface, and then use induction with a new $S$, namely the pre-image by $\varphi$ of such a hypersurface.

We shall use the lemmas below, for which we introduce a definition: given a rational function $f \in \overline{\mathbb{Q}}(x)$ its height will be the height of the vector of the coefficients of the defining polynomials. This height is naturally defined also for rational functions on curves, 
once $x$ is chosen and the function field of the curve is viewed as a finite extension of $\overline{\mathbb{Q}}(x)$. We shall not use sharp estimates, but only inequalities up to a constant factor.

LEMMA 4.2. There is a $c_{3}>0$ depending only on $\sigma$ such that for large $M$ we have $\max _{i} \operatorname{deg} f_{i, \theta}^{(M)} \geq c_{3} M^{2}$ for all $\theta \in \mathbb{P}_{d-1}(\overline{\mathbb{Q}})$ outside a suitable proper Zariski-closed set depending on $M$.

Sketch of the proof of the Lemma. The canonical height $\hat{h}\left(\sigma_{\lambda}\right)$ is $>0$, where this height is referred to the function field $\overline{\mathbb{Q}}\left(\mathcal{C}_{\lambda}\right)$. Hence there exists $c_{4}>0$ with $\hat{h}\left(M \sigma_{\lambda}\right)>c_{4} M^{2}$ for all $M \geq 0$. By the usual average procedure of Néron-Tate used to construct the canonical height from the naive height (see $\S 3$, page 29 in Serre's book [19]), as in the proof of Zimmer's theorem, applied to $\mathcal{A} \rightarrow \mathcal{C}_{\lambda}$ over the function field $\overline{\mathbb{Q}}\left(\mathcal{C}_{\lambda}\right)$, we deduce that $\max _{i}\left(\operatorname{deg} f_{i, \lambda}^{(M)}\right)>c_{5} M^{2}$. However, the special degree of a rational function in a family may decrease with respect to the generic one only in a proper Zariski closed set, proving the lemma.

Lemma 4.3. Let $\mathcal{C}_{\lambda}$, be as above defined over a number field $k, f_{\lambda} \in k\left(\mathcal{C}_{\lambda}\right)$ be a rational function of degree $d$. Let $h: S(\overline{\mathbb{Q}}) \rightarrow \mathbb{R}$ be a height function associated to an ample divisor; let $\delta$ be the degree of such divisor on the curve $\mathcal{C}_{\lambda}$. Then there exists a function $c_{5}=c_{5}\left(\epsilon, \operatorname{deg} f_{\theta}\right)$ such that for all $\epsilon>0$ and for all $\theta \in \mathbb{P}_{d-1}(\overline{\mathbb{Q}})$ for which $\mathcal{C}_{\theta}$ is irreducible and $f_{\theta}$ is defined,

$\left(\operatorname{deg} f_{\theta}-\epsilon\right) h(p)-c_{5} \cdot\left(h\left(f_{\theta}\right)+h(\theta)+1\right) \leq \frac{h\left(f_{\theta}(p)\right)}{\delta} \leq\left(\operatorname{deg} f_{\theta}+\epsilon\right) h(p)+c_{5} \cdot\left(h\left(f_{\theta}\right)+h(\theta)+1\right)$.

Remark: A proof should follow from the classical height machine, since the precise dependence of $c_{5}$ is not specified. Moreover, for our purpose it is sufficient to use the left inequality with $\operatorname{deg}(f) / 2$ instead of $\operatorname{deg}(f)-\epsilon$. In any case, a formal proof of the lemma as stated here follows for instance from Abouzaid's Corollary 1.2 of [1] as follows: we take a fixed rational function $x$ on $\mathcal{C}_{\lambda}$ and we apply Abouzaid's result to $F(\lambda, x, y) \in \overline{\mathbb{Q}}(\lambda)[x, y]$, the minimal polynomial such that $F\left(\lambda, x, f_{\lambda}\right)=0$. It is irreducible also as a polynomial over $\overline{\mathbb{Q}(\lambda)}$, since $\mathcal{C}_{\lambda}$ is absolutely irreducible over $\overline{\mathbb{Q}}(\lambda)$.

The lemma will be applied with $f_{\lambda}=f_{i, \lambda}^{(M)}$.

In the following lemma we bound the height of the coefficients of $f_{i, \lambda}^{(M)}$ :

LEMMA 4.4. In the above notation, there exists a function $M \mapsto c_{7}(M)$ (depending only on $\mathcal{A} \rightarrow S$ and $\sigma)$, such that for all $i=0, \ldots, N, \theta \in \mathbb{P}_{d-1}(\overline{\mathbb{Q}})$ and $M \geq 1$,

$$
h\left(f_{i, \theta}^{(M)}\right) \leq c_{7}(M) \cdot(1+h(\theta)) .
$$


Remark: We omit the easy proof. We could obtain $c_{7}(M)$ to be $c_{7} \cdot M^{2}$ but we shall not need this.

Let $\hat{h}_{\mathcal{A}_{s}}$ denote the Néron-Tate height in $\mathcal{A}_{s}$ associated to the above embedding (i.e. the renormalization of the naive height associated to the embedding) and $h_{\mathcal{A}}$ the naive height on $\mathcal{A}$, still relative to the given embedding; by restriction this gives the naive heights on $\mathcal{A}_{s}$. Then, with the above setting:

Lemma 4.5. There exist $\gamma_{1}, \gamma_{2}>0$ such that for all $s \in S$ and all $P \in \pi^{-1}(s)$,

$$
\left|\hat{h}_{\mathcal{A}_{s}}(P)-h_{\mathcal{A}}(P)\right| \leq \gamma_{1} h(s)+\gamma_{2} .
$$

This may be viewed as a 'Uniform Zimmer's Theorem' and essentially follows from Theorem 1.3, due to Silverman-Tate, Chapter 12 in Lang's book [9]. Note that we need the estimate only above a Zariski-dense subset $S_{0}$ of $S$.

We now proceed to the proof of the Theorem. As remarked before the above lemmas it suffices to prove the statement for all $\theta$ outside any prescribed hypersurface, because then we may argue by induction on the dimension of $S$, replacing $S$ with the inverse image $\varphi^{-1}$ (hypersurface).

Let $M$ be a sufficiently large integer to justify the estimate which shall follow, and let us argue supposing tacitly that $\theta$ does not belong to the above mentioned hypersurface, so that we can apply Lemma 4.2 to $\theta$.

Let then $\theta$ be such that $\mathcal{C}_{\theta}$ is non-torsion relative to $\sigma$. Suppose $p \in \mathcal{C}_{\theta}(\overline{\mathbb{Q}})$ is a point such that $\sigma(p)$ is torsion.

Then $\hat{h}_{\mathcal{A}_{p}}(\sigma(p))=0$ and also $\hat{h}_{\mathcal{A}_{p}}(M \sigma(p))=0$. By the last lemma,

$$
\mid h_{\mathcal{A}}\left(M \sigma(p) \mid \leq \gamma_{1} h(p)+\gamma_{2} .\right.
$$

However $M \sigma(p)=\left(1: f_{1, \theta}^{(M)}(p): \ldots: f_{N, \theta}^{(M)}(p)\right)$. By Lemma 4.4 (left inequality), Lemma 4.3 and Lemma 4.5 we obtain

$$
h_{\mathcal{A}}(M \sigma(p)) \geq c_{8} M^{2} h(p)-c_{9}(M) \cdot(1+h(\theta)) .
$$

for a function $c_{9}$, which can be suppose to be increasing in $M$.

Let us choose an index $i$ providing the maximum of the degree for $f_{i, \lambda}^{(M)}$. Comparing upper and lower bounds for $h(M \sigma(p))$ and expressing in term of $f_{i, \lambda}^{(M)}(p)$ we obtain

$$
h(p)\left(c_{8} M^{2}-\gamma_{1}\right) \leq \gamma_{2}+c_{9}(M)(1+h(\theta))
$$

Choose $M$ such that $c_{8} M^{2}>2 \gamma_{1}$ and we obtain for a suitable constant $C_{1}, h(p)<$ $C_{1}(h(\theta)+1)$. 


\section{APPENDIX B: Remarks on the split case}

In this Appendix, we shall deal with a very special case of our theorems when the family is not simple. This case has been treated in the paper [16], by methods somewhat complicated. Hence we believe it is not entirely free of interest to present here an alternative approach more in line with the methods used in this paper for the case of simple families.

For these reasons, we limit ourself to a very special situation, giving moreover only a sketch of the argument.

Let $S$ be a surface with algebraically independent regular functions $\lambda$, $\mu$, so that we have a generically surjective map $(\lambda, \mu): S \rightarrow \mathbb{A}^{2}$.

Our abelian family will be $\mathcal{A}_{s}: E_{\lambda(s)} \times E_{\mu(s)}$, where $E_{t}$ is the Legendre curve:

$$
E_{t}: y^{2}=x(x-1)(x-t)
$$

We tacitly assume that $\lambda, \mu$ do not assume in $S$ the value 0,1 . Now the section $\sigma: S \rightarrow \mathcal{A}$ corresponds to a pair of sections $P=P_{s} \in E_{\lambda(s)}, Q=Q_{s} \in E_{\mu(s)}$, where the coordinates of $P, Q$ (which may be viewed as points defined over a fucntion field) are rational functions of s. We suppose that none of $P, Q$ is torsion, which yields that they are linearly independent over $\operatorname{End}(\mathcal{A} / S)$, since $E_{\lambda}, E_{\mu}$ are not identically isogenous.

As for the general case treated in the paper, we can define the Betti coordinates of the section, for which we adopt the same notation. Now our proof considers the two cases, according to the rank of the differential of the Betti map. We notice at once that the generic rank can be two or four, since by Proposition 1.1 it must be even, and it can be zero only for a torsion section.

Case I. The generic rank of the differential of the Betti map is 2 .

Note that, writing the Betti map as $s \mapsto\left(a_{1}(s), a_{2}(s), a_{3}(s), a_{4}(s)\right)$, where $\left(a_{1}, a_{2}\right)$ is the Betti map corresponding to the first elliptic curve, $a_{1}, a_{2}$ (resp. $a_{3}, a_{4}$ ) must be analytically independent, for otherwise the fibers of the Betti map would have dimension $\leq 1$, hence 0 which we have excluded. Then locally either $a_{3}, a_{4}$ are functions of $a_{1}, a_{2}$ or vice-versa.

We let $U$ be the maximal open set where $\mathfrak{b}$ has rank exactly two. It is the complement of the set where the rank is zero; on this latter set the Betti coordinates are locally constant, so this set is a countable union of fibers for the Betti maps; as observed in the preliminaries, the fibers of the Betti maps are complex-analytic curves. So $U$ is the complement of an analytic curve.

As in the opening section of the paper, $U$ is the union of finitely many simply connected open sets $U_{\alpha}$. 
Lemma 5.1. For every $U_{\alpha}$ there exists a non empty open set $V_{\alpha} \subset U_{\alpha}$ and a 'transverse' algebraic curve $Z_{\alpha}$ defined over $\overline{\mathbb{Q}}$ that meets in $V_{\alpha}$ all the level curves which meet $V_{\alpha}$.

By 'transverse' we mean transverse in the usual sense, i.e. non-tangent, to the level curves of the Betti map.

Proof. As above, by restricting $U_{\alpha}$ we can suppose that two coordinates of the Betti map, say $a_{3}, a_{4}$, are functions of $a_{1}, a_{2}$ (see also the notation above). The map $s \mapsto$ $\left(a_{1}(s), a_{2}(s)\right)$ is injective with differential of maximal rank (so $a_{1}, a_{2}$ become coordinates in the image). The level curves above $a_{1}, a_{2}$ in $\left(U_{\alpha}\right)$ are level curves for $\left(a_{1}, a_{2}, a_{3}, a_{4}\right)$, therefore are complex-analytic; also, they are smooth because the rank of the differential is constant in $U_{\alpha}$.

Take an algebraic point $p$ in $U_{\alpha}$; it is a smooth point in the corresponding level curve. We now take an algebraic curve $Z_{\alpha}$, defined over $\overline{\mathbb{Q}}$, passing through $p$ and not tangent to the level curve. Shrinking the neighborhood of $p$, by the implicit function theorem again, we obtain the assertion.

Let us now fix the neighborhood $V_{\alpha}$ as in the lemma and let $Z_{\alpha}$ be the algebraic curve appearing in the lemma. By the construction of the lemma, the Betti map restricted to $Z_{\alpha}$ still has rank 2 As remarked above, we may suppose that the projection to the first two coordinates of the Betti map has still rank 2. Then we may find on $Z_{\alpha}$ a $z$ where the Betti coordinates $a_{1}, a_{2}$ are rational of any given large enough denominator and this point $z$ will be necessarily algebraic. This means that the section $P$ takes a torsion value $P_{z}$ on $E_{\lambda(z)}$, whose order is the said denominator. The point $z$ defines a level curve $X_{z}$ (the level curve containing $z$ ). This is a torsion curve for $P$, hence it is algebraic. Since $a_{3}, a_{4}$ are constant on $X_{z}$, by (a very special case of) Manin's Theorem of the kernel they must be rational. Then we obtain that for every point $z \in Z_{\alpha}$ which is torsion for the section $P$, this point is also torsion for the section $Q$. In this way we obtain infinitely many torsion values of the section $\sigma$ restricted to $Z$. By the algebraic case of [13], this is impossible since the restriction $\sigma_{Z_{\alpha}}$ is non torsion unless $P, Q$ are linearly dependent on $Z_{\alpha}$. However by Silverman's specialization theorem we may choose the curve $Z_{\alpha}$ in the lemma, with tangent vector of sufficiently large height so that this does not happen (or else, it suffices to exclude that $E_{\lambda}, E_{\mu}$ become isogenous along $Z_{\alpha}$; this can be achieved e.g. by taking $Z_{\alpha}$ to be a line in the plane $\lambda, \mu$, since only finitely many lines might correspond to isogenous families).

Case II: The generic rank of the differential is 4. Let us fix a transcendental $\lambda_{0}$ and consider the curve $V=V_{\lambda_{0}} \subset S$, defined by $\lambda=\lambda_{0}$. There exists a non-empty open subset (in the complex-topology) $A \subset E_{\lambda_{0}}$ such that $P^{-1}(A) \cap V_{\lambda_{0}}$ does not contain critical points 
of the jacobian. Let $X$ be a torsion curve of high order $N$. It intersects $V_{\lambda_{0}}$ in a point $x \in S$ because $\lambda_{0}$ is transcendental. Since $\lambda_{0}$ is transcendental, we may view $x$ as a $\overline{\mathbb{Q}}$-generic point of $X$. By Galois, if the order of torsion $N$ is sufficiently large, there is a conjugate of $x$ inside our open set $P^{-1}(A)$, so the open set $P^{-1}(A)$ would contain a level curve.

\section{APPENDIX C: An issue of Mazur}

Let us recall the last problem considered in the introduction. We take for the base $S$ a finite cover of the moduli space $M_{2}$ of (complex) curves of genus 2, which is a quasi projective algebraic variety of dimension three. The fiber $\mathcal{A}_{x}$ over a point $x \in M_{2}$ (representing a curve of genus 2) will be the jacobian of that curve, which is a principally polarized abelian surface. We thus obtain an abelian scheme of relative dimension 2 over a three-dimensional base.

Take now a non-torsion section $\sigma: S \rightarrow \mathcal{A}$. We shall prove

THEOREM 6.1. There exist torsion curves for $\sigma$ of arbitrarily high torsion order, and the union of these curves is topologically dense in $S$, and thus Zariski-dense.

Similarly to the proof of the Main Theorem, the present proof involves the rank of the Betti map, and we can split it into two cases according. Since this rank is even by Proposition 1.1, and since it cannot be zero (by Manin's theorem, because $\sigma$ is not torsion) we have only two cases to consider.

Case I. The generic rank of the differential of the Betti map is 4 (maximal rank).

In this case the Betti map is locally surjective, so in particular it takes some rational value in every open set of $S$. Also, on a suitable open dense subset of $S$, the rank is exactly 4 and there the fibers of the Betti map are complex subvarieties of dimension 1 (use Proposition 1.2 above). The fibers of the rational points, being torsion curves are algebraic curves. This proves our assertion in this case.

Case II. The generic rank of the differential of the Betti map is 2 (degenerate Betti map).

We shall in fact exclude this case, thus completing the proof of Theorem 6.1.

In this Case II, all non-empty fibers of the Betti map, which are complex varieties, will have complex dimension at least 2 (use again Proposition 1.2 above). On the other hand, by Manin's theorem, this dimension cannot be 3 (for otherwise the Betti map would be constant, hence a rational constant by Manin's theorem, so $\sigma$ would be torsion), so it will be exactly 2. If now the Betti map takes rational values with infinitely many denominators, we can apply our Main Theorem obtaining a contradiction. To prove that 
this would be in fact the case, we shall restrict our analysis to some special surfaces inside $M_{2}$, namely those corresponding to split-jacobians.

Let us first prove that there are infinitely many such surfaces. Given a (smooth, projective) genus-1 curve $E$ and two points $P_{1}, P_{2}$ on it, one can find, for every $d \geq 3$, a cover $X \rightarrow E$ of degree $d$, ramified only over $P_{1}, P_{2}$, where $X$ has genus 2 : it suffices to apply Riemann's Existence Theorem with the suitable combinatorial data, which in this case correspond to having exactly one ramified point over each $P_{i}, i=1,2$, with ramification index 2. Now, such curves $X$ depend, up to isomorphisms, on two parameters: the choice of the elliptic curve $E$ (one-dimensional moduli space) and the choice of the point $P_{2}-P_{1}$ (where the operation is made according to the group law on $E$ ). Hence we have a surface on the moduli space, and correspondingly a surface on $S$. Also, it is easy to see that if we take pairwise coprime values of the degree $d$, the corresponding surfaces are indeed distinct (here we use the fact that the Jacobian of a generic curve $X$ corresponding to a point on this surface is isogenous to a product of two non-isogenous elliptic curves, for otherwise the moduli space of such jacobians would be one-dimensional).

Now, by our Main Theorem applied to $S$, only finitely many such surfaces can be torsion surfaces.

Take one such surface which is not torsion; by our results in Appendix B, there are infinitely many torsion values for $\sigma$ on such a surface. This concludes the proof that Case II cannot occur.

As pointed out by the referee, in the situation of Appendix $\mathrm{C}$ the base, being a finite cover of the moduli space $\mathcal{A}_{2,1}$, admits an explicit compactification, where the points at infinity can be described in modular terms. This may perhaps be used to obtain a simpler proof, by 'algebraically' constructing the relevant curves $\mathcal{C}_{\theta}$ avoiding the torsion hypersurfaces at infinity.

\section{References}

[1] M. Abouzaid, Heights and logarithmic ged on algebraic curves, Int. Journal Number Theory 4 (2008), 177-197.

[2] Y. André, Mumford-Tate groups of mixed Hodge structures and the theorem of the fixed part, Compositio Mathematica 82 (1992), 1-24.

[3] Y. André, Groupes de Galois motiviques et périodes. Sém. Bourbaki, Exp. 1104, Nov. 2015.

[4] D. Bertrand, Galois descente in Galois theories. Sem. Congrès 23 (2011), 1-24. 
[5] E. Bierstone \& P. Milman, Semianalytic and Subanalytic Sets, Publications Math. I.H.E.S., 47 (1988), 5-42.

[6] S. David, Fonctions thêta et points de torsion des variétés abéliennes, Compositio Math., 78, n. 2 (1991), 121-160.

[7] P. Griffiths, J. Harris, A Poncelet theorem in space, Comm. Math. Helvetici 52 (1977), 145-160.

[8] Hormander, Complex Analysis in Several Varibales, North Holland, 1990.

[9] S. Lang, Fundamentals of Diophantine Geometry, Springer Verlag, 1983

[10] Lojasievicz, Introduction to Complex Analytic Geometry, Springer Verlag, 1991.

[11] Yu. Manin, Rational points on curves over function fields, Izv. Akad. Nauk SSSR 27 (1963), 1395-1490. English translation: Tansl. II Amer. Math. Soc. 50 (1966), 189-234.

[12] D. Masser, Specialization of endomorphism rings of abelian varieties, Bulletin de la S.M.F., 124, n.3 (1996), 457-476.

[13] D. Masser, U. Zannier, Torsion points on familes of squares of elliptic curves, Math. Annalen 352 (2012), 453-484.

[14] D. Masser, U. Zannier, Torsion points on familes of products of elliptic curves, Advances in Math. 259 (2014), 116-133.

[15] D. Masser, U. Zannier, Torsion points on families of simple abelian surfaces and Pell's equation over polynomial rings. With an appendix by E. V. Flynn, Journal E.M.S. (2015) 9, 2379-2416.

[16] D. Masser, U. Zannier, Torsion points on families of simple abelian varieties, Pell's equation and integration in elementary terms (in preparation).

[17] J. Pila, Rational points on a subanalytic surface, Ann. Inst. Fourier (Grenoble) 55, no. $5,1501-1516$.

[18] J-P. Serre, J. Tate, Good reduction of abelian varieties, Annals of Math. 88 (1968), 492-517. 
[19] J-P. Serre, Lectures on the Mordell-Weil theorem, Vieweg Verlag 1984.

[20] J-P. Serre, Quelques propriétés des groupes algébriques commutatifs, Appendix to: Nombres Transcendants et Groupes Algébriques, by M. Waldschmidt, Astérisque 69-70, Soc. Math. de France, 1987.

[21] J. Silverman, Heights and specialization map for families of abelian varieties, J. reine ang. Math. 342 (1983),197-211.

[22] P. Swinnerton-Dyer, Analytic theory of abelian varieties, Cambridge University Press, 1974.

[23] C. Voisin, Torsion points of sections of Lagrangian torus fibrations and the Chow ring of hyper-Kähler fourfolds, preprint 2017.

[24] U. Zannier, Some Problems of Unlikely Intersections in Arithmetic and Geometry, Princeton U. Press (2014).

Pietro Corvaja

Dipartimento di Scienze matematiche,

INFORMATICHE E FISICHE

UNIVERSITÀ DI UDINE

Via Delle Scienze, 206

33100 UDINE

ITALY

E-mail address: pietro.corvaja@uniud.it

DAVid Masser

Mathematisches Institut

UNIVERSITÄT BASEL

SPIEgElgasse 1 ,

CH-4051 BASEL

SwitzerLAND

E-mail address: david.masser@unibas.ch 
UMBERTO ZanNiER

Scuola Normale Superiore

Piazza deI Cavalieri, 7

56126 PISA

ITALY

E-mail address: u.zannier@sns.it 


\section{LATEST PREPRINTS}

No. Author: Title

2016-28Ｉ. Capuano, D. Masser, J. Pila, U. Zannier

Rational points on Grassmannians and unlikely intersections in tori

2016-29 C. Nobili, F. Otto

Limitations of the background field method applied to Rayleigh-Bénard convection

2016-30Ｗ. D. Brownawell, D. W. Masser

Unlikely intersections for curves in additive groups over positive characteristic

2016-31 M. Dambrine, H. Harbrecht, M. D. Peters, B. Puig

On Bernoulli's free boundary problem with a random boundary

2016-32 H. Harbrecht, J. Tausch

A fast sparse grid based space-time boundary element method for the nonstationary heat equation

2016-33 S. Iula

A note on the Moser-Trudinger inequality in Sobolev-Slobodeckij spaces in dimension one

2016-34 C. Bürli, H. Harbrecht, P. Odermatt, S. Sayasone, N. Chitnis Mathematical analysis of the transmission dynamics of the liver fluke, Opisthorchis viverrini

2017-01 J. Dölz and T. Gerig, M. Lüthi, H. Harbrecht and T. Vetter Efficient computation of low-rank Gaussian process models for surface and image registration

2017-02 M. J. Grote, M. Mehlin, S. A. Sauter

Convergence analysis of energy conserving explicit local time-stepping methods for the wave equation

2017-03 Y. Bilu, F. Luca, D. Masser

Collinear CM-points

2017-04 P. Zaspel

Ensemble Kalman filters for reliability estimation in perfusion inference

2017-05 J. Dölz and H. Harbrecht

Hierarchical Matrix Approximation for the Uncertainty Quantification of

Potentials on Random Domains

Preprints are available under https://math.unibas.ch/research/publications 


\section{LATEST PREPRINTS}

No. Author: Title

2017-07 H. Harbrecht and M. Schmidlin

Multilevel Methods for Uncertainty Quantification of Elliptic PDEs with Random Anisotropic Diffusion

2017-08 M. Griebel and H. Harbrecht

Singular value decomposition versus sparse grids: Refined complexity Estimates

2017-09 J. Garcke and I. Kalmykov

Efficient Higher Order Time Discretization Schemes for Hamilton-JacobiBellman Equations Based on Diagonally Implicit Symplectic Runge-Kutta Methods

2017-10 M. J. Grote and U. Nahum

Adaptive Eigenspace Regularization For Inverse Scattering Problems

2017-11 J. Dölz, H. Harbrecht, S. Kurz, S. Schöps and F. Wolf

A Fast Isogeometric BEM for the Three Dimensional Laplace- and

Helmholtz Problems

2017-12 P. Zaspel

Algorithmic patterns for $\mathcal{H}$-matrices on many-core processors

2017-13 R. Brügger, R. Croce and H. Harbrecht

Solving a free boundary problem with non-constant coefficients

2017-14 M. Dambrine, H. Harbrecht and B. Puig

Incorporating knowledge on the measurement noise in electrical impedance tomography

2017-15 C. Bürli, H. Harbrecht, P. Odermatt, S. Sayasone and N. Chitnis Analysis of Interventions against the Liver Fluke, Opisthorchis viverrini

2017-16 D. W. Masser

Abcological anecdotes

2017-17 P. Corvaja, D. W. Masser and U. Zannier

Torsion hypersurfaces on abelian schemes and Betti coordinates

Preprints are available under https://math.unibas.ch/research/publications 